MULTIVARIATE BEHAVIORAL RESEARCH, 42(1), 185-227

Copyright $\odot$ 2007, Lawrence Erlbaum Associates, Inc.

\title{
Addressing Moderated Mediation Hypotheses: Theory, Methods, and Prescriptions
}

\author{
Kristopher J. Preacher \\ University of Kansas \\ Derek D. Rucker \\ Northwestern University \\ Andrew F. Hayes \\ The Ohio State University
}

This article provides researchers with a guide to properly construe and conduct analyses of conditional indirect effects, commonly known as moderated mediation effects. We disentangle conflicting definitions of moderated mediation and describe approaches for estimating and testing a variety of hypotheses involving conditional indirect effects. We introduce standard errors for hypothesis testing and construction of confidence intervals in large samples but advocate that researchers use bootstrapping whenever possible. We also describe methods for probing significant conditional indirect effects by employing direct extensions of the simple slopes method and Johnson-Neyman technique for probing significant interactions. Finally, we provide an SPSS macro to facilitate the implementation of the recommended asymptotic and bootstrapping methods. We illustrate the application of these methods with an example drawn from the Michigan Study of Adolescent Life Transitions, showing that the indirect effect of intrinsic student interest on mathematics performance through teacher perceptions of talent is moderated by student math self-concept.

Correspondence concerning this article should be addressed to Kristopher J. Preacher, University of Kansas, Department of Psychology, 1415 Jayhawk Boulevard, Room 426, Lawrence, KS 660457556. E-mail: preacher@ku.edu 
Mediation, or an indirect effect, is said to occur when the causal effect of an independent variable $(X)$ on a dependent variable $(Y)$ is transmitted by a mediator $(M)$. In other words, $X$ affects $Y$ because $X$ affects $M$, and $M$, in turn, affects $Y$. Mediation effect and indirect effect are often used interchangeably (as they are here), although some authors have drawn distinctions between them (e.g., Holmbeck, 1997). Methods to assess mediation became particularly popular in psychology after publications by Judd and Kenny (1981) and Baron and Kenny (1986). Today, examples of this simple type of mediation effect are so numerous that one can open an issue of virtually any major social science journal and find at least one test of mediation. For example, Fredrickson, Tugade, Waugh, and Larkin (2003) hypothesized that positive emotions mediate the effect of psychological resilience on residual resources (life satisfaction, optimism, and tranquility). Calvete and Cardenoso (2005) demonstrated that the effect of gender on depressive symptoms is mediated by need for acceptance, positive thinking, self-focused negative cognitions, and negative problem orientation. Hundreds of new mediation hypotheses are proposed and tested in the literature every year. In response to high demand for appropriate methods, a large literature now exists that details methods by which mediation may be assessed in models of ever-increasing complexity.

It is often of critical interest to determine whether or not a mediation effect remains constant across different contexts, groups of individuals, and values of the independent variable. For example, perhaps $M$ mediates the $X \rightarrow Y$ relationship for boys but not for girls. More generally, the strength of an indirect effect may depend linearly upon the value of a moderator $(W)$ that is measured on an interval or ratio scale. Of course, testing such additional hypotheses requires the development of appropriate statistical tests. In recognition of this requirement, this article aims to educate and help researchers with regard to how to analyze indirect effects that depend on other variables in the model under scrutiny. There are several ways in which hypotheses combining mediation and moderation may be modeled. Various sources refer to some of these effects as mediated moderation or moderated mediation (e.g., Baron \& Kenny, 1986), but there is a fair amount of confusion over precisely what pattern of causal relationships constitutes each kind of effect and how to assess the presence, strength, and significance of these effects. For simplicity, we gather such effects under the general rubric conditional indirect effects. We define a conditional indirect effect as the magnitude of an indirect effect at a particular value of a moderator (or at particular values of more than one moderator).

Examples of conditional indirect effect hypotheses are common in the literature. For example, the mediation effects found by Calvete and Cardenoso (2005) mentioned previously were further hypothesized to be moderated by age. Although studies investigating mediation, moderation, or both are abundant, formal tests of conditional indirect effects are less common. We surmise that conditional 
indirect effects may be relevant and interesting in many settings, but generally may go unnoticed and unexamined because clear methods have not yet been articulated in the literature for investigating whether (and, if so, how) an indirect effect varies systematically as a function of another variable. In addition to introducing methods that can be used to investigate conditional indirect effects, we illustrate these methods using an example drawn from the Michigan Study of Adolescent Life Transitions (MSALT). Specifically, we show how the indirect effect of intrinsic student interest in math (the independent variable) on mathematics performance (the dependent variable) through teacher perceptions of talent (a mediator) is moderated by student math self-concept.

\section{OVERVIEW OF OBJECTIVES}

We have several objectives in this article. First, consistent with recent efforts to disentangle confusion over moderated mediation (e.g., Muller, Judd, \& Yzerbyt, 2005), we provide a guide to help resolve the confusion that persists in the literature regarding conflicting definitions of moderated mediation. Second, as called for by Muller et al. (2005), we provide intuitive approaches for testing hypotheses of conditional indirect effects. To this end, we introduce standard errors $(S E s)$ for various conditional indirect effects and discuss the utility of bootstrapping and normal-theory methods. Third, we describe methods for probing moderated mediation effects by employing direct extensions of methods familiar to many researchers in the context of probing significant interactions. Specifically, we implement a direct extension of the simple slopes procedure (Aiken \& West, 1991) to probe moderated mediation effects. We also suggest that the regions of significance approach (or the Johnson-Neyman technique) be extended to probing moderated mediation effects, identifying ranges of the moderator for which an indirect effect is statistically significant. Finally, we provide an SPSS macro to facilitate the implementation of the recommended asymptotic and bootstrapping methods, illustrating its use with a real-world example. Our procedures are illustrated within a regression or path-analytic framework (with no latent variables), but our strategies can be easily applied in more complex structural equation models (SEMs). This article is aimed primarily at the applied researcher to whom the methods will be most useful, but there is also much that will be of interest to methodologists. The ultimate goal and contribution of this article is to offer researchers and practitioners an intuitive guide to construe and conduct complex mediation analyses involving conditional indirect effects.

Before discussing conditional indirect effects, we briefly review simple mediation and moderation and discuss methods traditionally used to investigate their presence. We then present methods for assessing the presence, strength, 
and significance of conditional indirect effects to facilitate the understanding of moderated mediation.

\section{SIMPLE MEDIATION}

Mediation analysis permits examination of process, allowing the researcher to investigate by what means $X$ exerts its effect on $Y$. Although systems of equations linking $X$ to $Y$ through multiple mediators are possible to specify (MacKinnon, 2000 ), we focus on models in which only a single mediator $(M)$ is posited. We term this three-variable system simple mediation. Simple mediation is illustrated in the path diagram in Figure 1. In the figure, $a_{1}$ refers to the (unstandardized) slope coefficient of $M$ regressed on $X$, and $b_{1}$ and $c^{\prime}$ denote the conditional coefficients of $Y$ regressed on $M$ and $X$, respectively, when both are included as simultaneous predictors of $Y$. Letting $c$ represent the effect of $X$ on $Y$ in the absence of $M$, the indirect effect is traditionally quantified as $c-c^{\prime}$, which is ordinarily equivalent to $a_{1} b_{1}$ (MacKinnon, Warsi, \& Dwyer, 1995).

The coefficients previously described are commonly obtained using leastsquares regression. Specifically, coefficients $a_{1}$ and $b_{1}$ may be obtained from the regression equations:

$$
\begin{aligned}
& M=a_{0}+a_{1} X+r \\
& Y=b_{0}+c^{\prime} X+b_{1} M+r
\end{aligned}
$$

where $a_{0}$ and $b_{0}$ are intercept terms and $r$ is a regression residual. The coefficients $a_{1}$ and $b_{1}$ are then used to assess the presence, strength, and significance of the indirect effect of $X$ on $Y$ via $M$. All of the models considered here

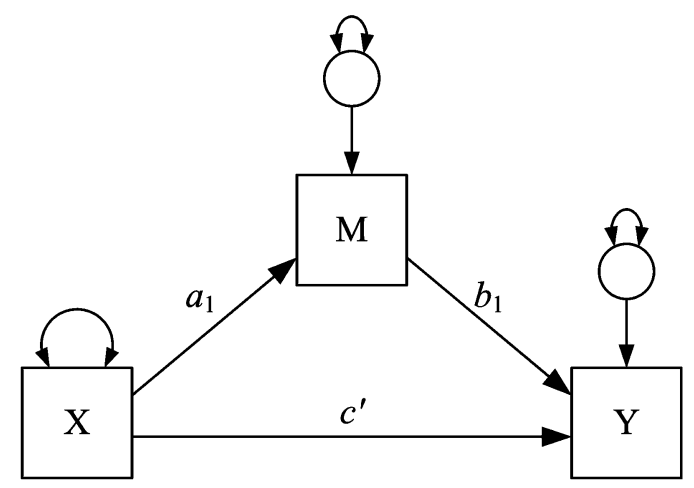

FIGURE 1 Simple mediation. 
may be assessed using SEM software or standard least-squares or maximum likelihood regression routines.

\section{ASSESSING THE PRESENCE, STRENGTH, AND SIGNIFICANCE OF INDIRECT EFFECTS}

MacKinnon and colleagues (MacKinnon, Lockwood, Hoffman, West, \& Sheets, 2002; MacKinnon, Lockwood, \& Williams, 2004) review a variety of strategies to gauge the extent and significance of indirect effects. The most popular of these strategies are the causal steps strategy, distribution of the product strategies, resampling or bootstrapping strategies, and various product of coefficients strategies. We do not dwell on all four approaches here. The causal steps strategy suffers from low power and does not directly address the hypothesis of interest (MacKinnon et al., 2002). Most methodologists agree that the product term $a_{1} b_{1}$, the quantity of interest in the remaining three strategies, is a proper quantification of the indirect effect. The distribution of the product strategy is probably the most accurate analytic method available for determining the significance of, and confidence intervals (CIs) for, $a_{1} b_{1}$ in simple mediation models (MacKinnon et al., 2004). However, extending this method to the study of conditional indirect effects will involve extensive analytic work and programming because the expressions for conditional indirect effects are more complex than those for simple mediation effects. We therefore limit our attention to the product of coefficients and bootstrapping strategies. We briefly explore each of these strategies in turn because each has implications for how conditional indirect effects can be appropriately assessed.

\section{Product of Coefficients Strategies}

An indirect effect is conceptualized as a population quantity that must be estimated in the sample. Sample indirect effects are quantified as products of sample estimates of regression coefficients. In the case of simple mediation, the point estimate of the indirect effect is $\hat{a}_{1} \hat{b}_{1}$, where the hat notation denotes a sample estimate of a population quantity. Under the assumptions of maximum likelihood and ordinary least squares, $\hat{a}_{1}$ and $\hat{b}_{1}$ are asymptotically independent and normally distributed. When it is further assumed that the product $\hat{a}_{1} \hat{b}_{1}$ is normally distributed, the exact $S E$ (Aroian, 1947; Craig, 1936; Goodman, 1960) is:

$$
S E_{\hat{a}_{1} \hat{b}_{1}}=\sqrt{\hat{a}_{1}^{2} s_{\hat{b}_{1}}^{2}+\hat{b}_{1}^{2} s_{\hat{a}_{1}}^{2}+s_{\hat{a}_{1}}^{2} s_{\hat{b}_{1}}^{2}}
$$


The result in Equation 3 can be obtained via the delta method using a secondorder Taylor series approximation (MacKinnon et al., 1995; Mood, Graybill, \& Boes, 1974, pp. 180, 534). Further discussion can be found in the Technical Appendix. In most cases, the $s_{\hat{a}_{1}}^{2} s_{\hat{b}_{1}}^{2}$ term is negligibly small and can be safely omitted, yielding:

$$
S E_{\hat{a}_{1} \hat{b}_{1}}=\sqrt{\hat{a}_{1}^{2} s_{\hat{b}_{1}}^{2}+\hat{b}_{1}^{2} s_{\hat{a}_{1}}^{2}}
$$

This approximation can also be obtained by implementing the first-order delta method (Sobel, 1982, 1986). These $S E$ s can be used in $z$-tests to determine the statistical significance of $\hat{a}_{1} \hat{b}_{1}$. They can also be used to construct $100(1-\alpha) \%$ confidence limits for the population product term $a_{1} b_{1}$ :

$$
\mathrm{CI}_{1-\alpha}: \hat{a}_{1} \hat{b}_{1} \pm z_{\alpha / 2} S E_{\hat{a}_{1} \hat{b}_{1}}
$$

One drawback associated with hypothesis tests or CIs using the product of coefficients strategy just described is that it requires the assumption that $\hat{a}_{1} \hat{b}_{1}$ is normally distributed. In fact, $\hat{a}_{1} \hat{b}_{1}$ is usually positively skewed and kurtotic (Bollen \& Stine, 1990; Lockwood \& MacKinnon, 1998; MacKinnon, Lockwood, \& Hoffman, 1998; Shrout \& Bolger, 2002; Stone \& Sobel, 1990). If the sample is large enough, $\hat{a}_{1} \hat{b}_{1}$ tends toward normality, but "large enough" by statisticians' standards is not always achieved in practice. If the product of coefficients strategy is used in significance testing or to form CIs, it is desirable that the total sample size $(N)$ be as large as possible to ensure good normal approximation. If the total sample size is not large, we recommend using bootstrapping, discussed next.

\section{Bootstrapping}

A growing literature now advocates the use of bootstrapping for assessing indirect effects (Bollen \& Stine, 1990; Lockwood \& MacKinnon, 1998; MacKinnon et al., 2004; Preacher \& Hayes, 2004; Shrout \& Bolger, 2002). Bootstrapping is one of several resampling strategies for estimation and hypothesis testing. In bootstrapping, the sample is conceptualized as a pseudo-population that represents the broader population from which the sample was derived, and the sampling distribution of any statistic can be generated by calculating the statistic of interest in multiple resamples of the data set. Using bootstrapping, no assumptions about the shape of the sampling distribution of the statistic are necessary when conducting inferential tests.

The sampling distribution of an indirect effect is estimated through bootstrapping by sampling $N$ units with replacement from the original sample of $N$ units. For each resample, $\hat{a}_{1}^{+} \hat{b}_{1}^{+}$is computed, where $\hat{a}_{1}^{+}$and $\hat{b}_{1}^{+}$are the analogues of 
$\hat{a}_{1}$ and $\hat{b}_{1}$ but computed in the resampled data set rather than the original sample. Repeated a total of $k$ times and then sorting the $k$ bootstrap values of $\hat{a}_{1}^{+} \hat{b}_{1}^{+}$ from low to high, the upper and lower bounds of a $100(1-\alpha) \%$ CI for $a_{1} b_{1}$ is defined as the $(\alpha / 2) k$ th and $(1+(1-\alpha / 2) k)$ th values in this sorted distribution. Because no symmetry assumption is made about the sampling distribution, the CI can be (and often is) asymmetric in accordance with the skewness of the sampling distribution of $\hat{a}_{1} \hat{b}_{1}$. For hypothesis testing, the null hypothesis of no indirect effect is rejected at the $\alpha$ level of significance if 0 lies outside the CI. Such percentile-based CIs can be further improved through bias-correction or bias-correction and acceleration. These bias correction methods work by adjusting the ordinal positions of the $\hat{a}_{1}^{+} \hat{b}_{1}^{+}$values in the sorted distribution of $\hat{a}_{1}^{+} \hat{b}_{1}^{+}$that are used as the upper and lower bounds of the confidence interval. MacKinnon et al. (2004) showed that such corrections can improve CIs and inferences when used in the context of simple mediation models. For the complex computational details of these corrections to percentile CIs, see Efron (1987), Efron and Tibshirani (1998), Lunneborg (2000), Preacher and Hayes (2006), or Stine (1989).

Only minor drawbacks are associated with bootstrapping. First, computation of the confidence limits is more time-consuming than in single-sample methods. But with the increasing speed of computer processors, speed is no longer a serious limitation, and some statistical analysis programs have implemented bootstrapping methodologies. Those that have not often can be programmed to do so (e.g., Lockwood \& MacKinnon, 1998; Preacher \& Hayes, 2004; Shrout \& Bolger, 2002). Thus, bootstrapping is more feasible now than in the past. Second, bootstrapping yields slightly different CIs each time the method is applied to the same data. Although it is true that different sets of $k$ bootstrap resamples will yield different estimates, the variation due to random resampling diminishes as $k$ increases.

In what follows, we extend the product of coefficients strategy and describe in more detail how bootstrapping can be used to estimate conditional indirect effects. But first, we provide an overview of methods used to investigate moderation.

\section{MODERATION}

When the strength of the relationship between two variables is dependent on a third variable, moderation is said to be occurring. The third variable, or moderator $(W)$, interacts with $X$ in predicting $Y$ if the regression weight of $Y$ on $X$ varies as a function of $W$. Moderation is typically assessed with the regression equation:

$$
Y=a_{0}+a_{1} X+a_{2} W+a_{3} X W+r,
$$


where $W$ is considered the moderator. Equation 6 may be reexpressed as

$$
Y=\left(a_{0}+a_{2} W\right)+\left(a_{1}+a_{3} W\right) X+r,
$$

clarifying how the simple slope of $Y$ regressed on $X,\left(a_{1}+a_{3} W\right)$, is a function of the moderator.

If $\hat{a}_{3}$ is significant, the interaction effect may be probed to determine whether the simple slope of $Y$ on $X$ is statistically significant for chosen conditional values of $W$ (typically the mean and \pm 1 SD from the mean for continuous $W$, and coded values for dichotomous $W$ ). This approach is described in detail by Aiken and West (1991). The quantity $\left(\hat{a}_{1}+\hat{a}_{3} W\right)$ may be divided by its $S E$ to yield a critical ratio test statistic distributed as $t$ with $d f=N-q$ in small samples (where $q$ is the number of estimated regression coefficients), or $z$ in large samples. The $S E$ of the simple slope is:

$$
S E_{\left(\hat{a}_{1}+\hat{a}_{3} W\right)}=\sqrt{s_{\hat{a}_{1}}^{2}+2 s_{\hat{a}_{1} \hat{a}_{3}} W+s_{\hat{a}_{3}}^{2} W^{2}}
$$

The simple regressions of $Y$ on $X$ at conditional values of $W$ are also typically plotted to facilitate interpretation.

Rather than choosing a limited number of arbitrary conditional values of $W$ and investigating the significance of the simple slopes at those values, we can instead seek the values of $W$ for which the simple slope of $Y$ regressed on $X$ is significant (Johnson \& Neyman, 1936). The result is a region of significance, or a range of values of $W$ for which $\left(\hat{a}_{1}+\hat{a}_{3} W\right)$ is significantly different from zero. We refer to this strategy as the Johnson-Neyman (J-N) technique after its originators. Continuously plotted CIs around simple slopes for all values of $W$ are termed confidence bands ${ }^{1}$ (Bauer \& Curran, 2005; Preacher, Curran, \& Bauer, 2006; Rogosa, 1980, 1981). $W$ moderates the relationship between $X$ and $Y$ for values of $W$ where the confidence bands do not contain zero. Confidence bands can easily be plotted to facilitate the interpretation of interaction effects (Preacher, Curran, \& Bauer, 2003, 2006). We extend simple slopes analysis, the J-N technique, and Rogosa's $(1980,1981)$ confidence bands method to the analysis of conditional indirect effects.

\footnotetext{
${ }^{1}$ There is a distinction between simultaneous and nonsimultaneous confidence bands and regions of significance (Pothoff, 1964). For nonsimultaneous bands, rejection rates are accurate for any given conditional value of the moderator. Simultaneous bands, on the other hand, describe regions of the moderator for which the simple slope will be significant for all values of the moderator $100(1-\alpha) \%$ of the time, in the long run. Only nonsimultaneous confidence bands and regions of significance are discussed here, as we consider them more practically relevant. For more information on the distinction between simultaneous and nonsimultaneous bands, consult Pothoff (1964) and Rogosa (1981).
} 


\section{MODERATED MEDIATION}

In both the applied literature and in discussions with colleagues, we have observed considerable confusion over what effects should be described as mediated moderation vs. moderated mediation and how to properly assess them. Baron and Kenny (1986) described a generally agreed-upon method for assessing mediated moderation (a term they coined) that involves first showing an interaction effect of $X$ and $W$ on $Y$, then introducing a mediator of that effect. In such models researchers may be interested in probing the interaction effects of $X$ and $W$ on $M$ and on $Y$ separately to clarify the nature of key relationships. However, because mediated moderation does not require the probing of conditional indirect effects, we do not further consider mediated moderation, but focus instead on moderated mediation.

James and Brett (1984) coined the term moderated mediation, suggesting it for mediation models involving relations that "require the addition of a moderator for either the $\hat{m}=f(x)$ or $\hat{y}=f(m)$ relations, or both" (p. 314). Moderated mediation models attempt to explain both how and when a given effect occurs (Frone, 1999). Formally, moderated mediation occurs when the strength of an indirect effect depends on the level of some variable, or in other words, when mediation relations are contingent on the level of a moderator.

There are multiple ways in which the magnitude of an indirect effect may be dependent upon a moderator. We enumerate several specific ways to think about moderated mediation effects, which we refer to as Models 1 through 5. Relying on the symbolic representation of mediation in Figure 1,

1. The independent variable $(X)$ functions as a moderator of the $b_{1}$ path.

2. Some fourth variable $(W)$ affects the $a_{1}$ path.

3. $W$ affects the $b_{1}$ path.

4. $W$ affects $a_{1}$ whereas yet another variable $(Z)$ affects $b_{1}$.

5. $W$ affects both $a_{1}$ and $b_{1}$.

These possibilities are presented in formal path diagrams in Figure 2. This list does not exhaust the possibilities but contains models we have encountered in the literature and helps to illustrate a framework within which to discuss possible ways to address these and similar hypotheses.

Several methodologists have defined or discussed moderated mediation, sometimes with conflicting definitions. Baron and Kenny (1986) offer an example of moderated mediation that coincides with our Model 5, using a causal steps strategy to gauge the presence of an indirect effect. Wegener and Fabrigar (2000) share James and Brett's (1984) definition: "Moderated mediation could occur when a moderator $\times$ IV interaction is observed (because of differences in IV to mediator and/or mediator to DV paths) or when no moderator $\times$ IV interaction is observed (because different mediators create the same magnitude of 


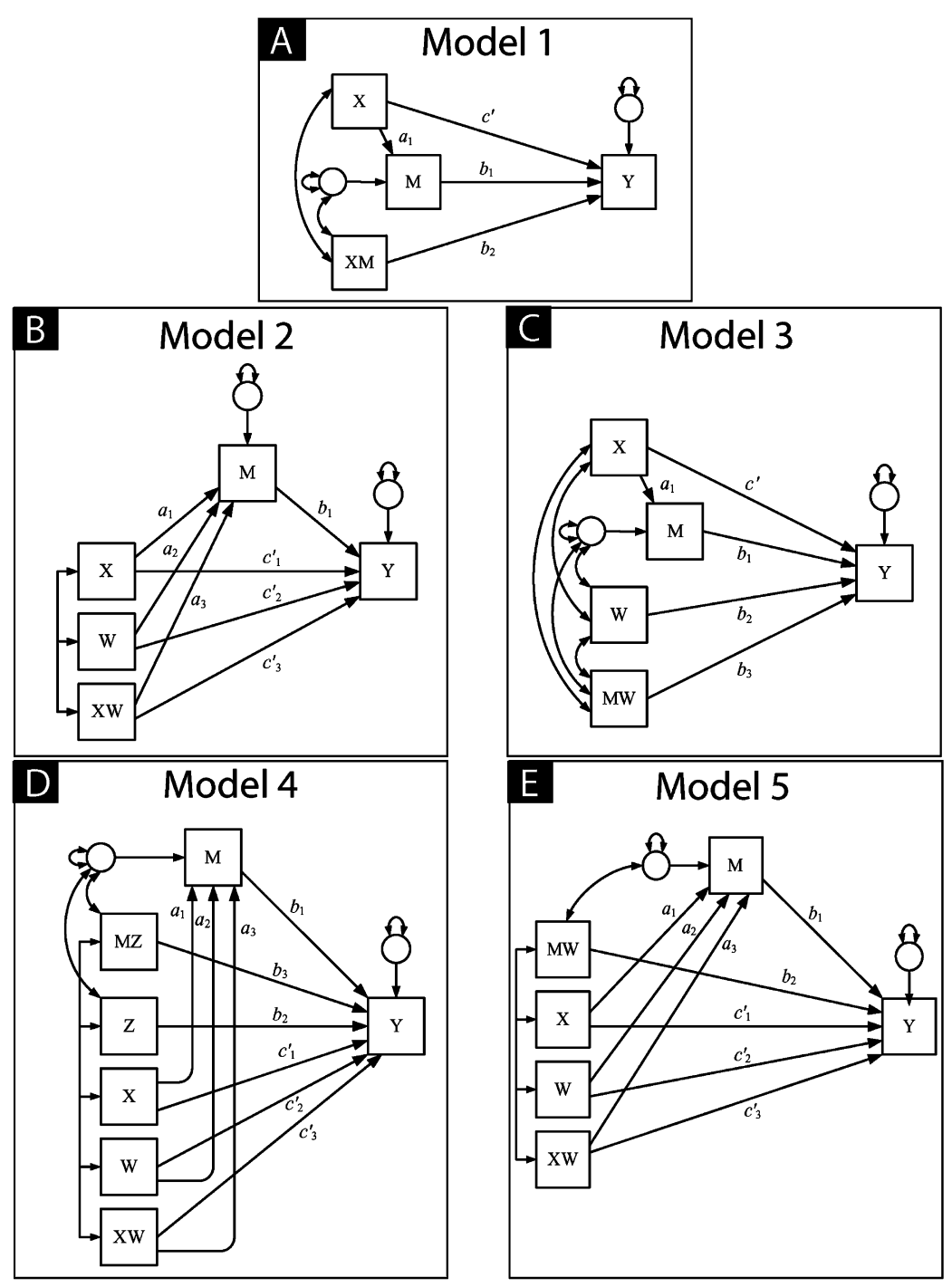

FIGURE 2 Models 1 through 5 represented as path diagrams.

effect or a mediator operates at some levels of the moderator but direct effects occur at other levels)" (p. 437), roughly coinciding with our Models 1, 2, and 3. Morgan-Lopez and MacKinnon (2006) characterize moderated mediation as the case when "the path from the intervention to the mediator (i.e., $X \rightarrow M$ ) is constant, whereas the effect of the mediator on the outcome (i.e., $M \rightarrow Y$ ) 
depends on the level of $Z$ " (p. 78), or our Model 3. Muller et al. (2005) asserted that moderated mediation "happens if the mediating process that is responsible for producing the effect of the treatment on the outcome depends on the value of a moderator variable," encompassing our Models 2, 3, 4, and 5. A similar definition is given by Rose, Holmbeck, Coakley, and Franks (2004).

We address conflicting definitions of moderated mediation by including all these as examples of the more general phenomenon of systematic variation in conditional indirect effects. We believe this choice is warranted because all of the effects described above represent mediation effects that vary in strength conditional on the value of at least one moderator variable. Because the strength of a simple mediation effect is quantified by $a_{1} b_{1}$, any moderation of this quantity by a moderator, by definition, results in an indirect effect that is conditional on some other variable.

In models like Models 1 to 5, the conditional indirect effect may be probed for significance using methods directly analogous to those used to probe significant interaction effects in regression (Aiken \& West, 1991; Morgan-Lopez, 2003; Muller et al., 2005; Tein, Sandler, MacKinnon, \& Wolchik, 2004). This method requires that the researcher have in mind a few values of the moderator for which it would be meaningful to examine the magnitude and significance of the indirect effect. Muller et al. (2005) and Tein et al. (2004), in a clever extension of a procedure described by Aiken and West (1991), Darlington (1990), and Judd and McClelland (1989), further outline a procedure whereby the researcher may center the moderator at conditional values and use key regression weights to interpret mediation effects as if the model were a simple mediation model. Our extension of the J-N technique to conditional indirect effects has the advantage that it does not require choosing possibly arbitrary conditional values. We now discuss how the conditional indirect effect in each of the numbered models in Figure 2 may be quantified in terms of sample point estimates. We follow this discussion with two methods for testing hypotheses using these point estimates: bootstrapping and an extension of the product of coefficients approach.

\section{Model 1: When the Independent Variable Is Also the Moderator}

Panel A in Figure 2 depicts the case in which the effect of $M$ on $Y$ is moderated by the independent variable $X$. Such models were described by Judd and Kenny (1981) and were presented as one example of moderated mediation by James and Brett (1984). ${ }^{2}$ Often a variable $M$ is investigated to determine whether it

\footnotetext{
${ }^{2}$ This model can also be understood as one in which the path linking $X$ to $Y\left(c^{\prime}\right)$ is moderated by $M$. The importance of considering such models in the context of program development is discussed by Judd, Kenny, and McClelland (2001) and MacKinnon (2001).
} 
serves as a mediator, a moderator, or both. For example, Lambert et al. (2003) investigated the role of state anxiety as a mediator and/or moderator of the effect of context (private vs. public) on cognitive control over prejudiced reactions using separate mediation and moderation analyses. Such investigations could theoretically be conducted on the basis of a single path analysis. The relevant regression equations for this type of conditional indirect effect are:

$$
\begin{aligned}
M & =a_{0}+a_{1} X+r \\
Y & =b_{0}+c^{\prime} X+\left(b_{1}+b_{2} X\right) M+r
\end{aligned}
$$

Equation 10 clarifies how the regression of $Y$ on $M$ can be considered conditional on $X$.

The conditional indirect effect of $X$ on $Y$ in Model 1 can be derived using an approach described by Sobel (1986) and Bollen (1987, 1989), which we discuss in detail in the Technical Appendix. The point estimate of the conditional indirect effect $^{3}$ of $X$ on $Y$ is in this case $f(\hat{\boldsymbol{\theta}} \mid X)=\hat{a}_{1}\left(\hat{b}_{1}+\hat{b}_{2} X\right)$. It is easy to see from the expression of $f(\hat{\boldsymbol{\theta}} \mid X)$ that the conditional indirect effect can depend on the chosen value of $X$. If the interaction effect between $X$ and $M$ is close to zero, then $\hat{b}_{2}$ will be close to zero, $X$ will have little influence on the indirect effect, and the conditional indirect effect reduces to $\hat{a}_{1} \hat{b}_{1}$ for all values of the moderator.

\section{Model 2: When the $a$ Path is Moderated by $W$}

Figure 2, Panel B depicts the model used to investigate two types of conditional indirect effect. These indirect effects are hypothesized when theory suggests that (1) a moderation effect is mediated by $M$ (an effect sometimes called mediated moderation, as discussed by Muller et al., 2005, among others) or (2) the $a_{1}$ path of an otherwise simple mediation model is moderated by $W$ (an effect traditionally termed moderated mediation). Paradoxically, the same model may be used to address either hypothesis, but different parameters are emphasized in each. Mediated moderation is addressed by examining the significance of the product $a_{3} b_{1}$. We restrict attention to the case when there exists an indirect effect of $X$ on $Y$ through $M$ (the simplest form of indirect effect), with the pertinent question regarding whether mediation exists for different conditional values of $W$. For example, Tein et al. (2004) examined whether self-efficacy mediated the effect of cultural norms on cigarette use differently depending on the number of peers who smoked. Hodges and Perry (1999) demonstrated

\footnotetext{
${ }^{3}$ Our notation denotes that the indirect effect is a function of the random variables (regression coefficients) contained in the vector $\hat{\boldsymbol{\theta}}$ evaluated at conditional values of the moderator(s) after the vertical bar.
} 
that children's internalizing and physical weakness influence physical and verbal victimization, and that victimization in turn influences future internalizing and peer rejection. They showed that the first of these effects is moderated by peer rejection.

Using methods exactly analogous to those used in the previous section (see Technical Appendix), this conditional indirect effect of $X$ on $Y$ may be expressed as $f(\hat{\boldsymbol{\theta}} \mid W)=\hat{b}_{1}\left(\hat{a}_{1}+\hat{a}_{3} W\right)$. Similar to Model 1 , the conditional indirect effect here depends on $W$ to the extent that the interaction coefficient $\hat{a}_{3}$ departs from zero.

\section{Model 3: When the $b$ Path Is Moderated by $W$}

Often the $b_{1}$ path of an otherwise simple mediation model is theorized to be moderated by another variable. The result is another process commonly referred to as moderated mediation. For example, Madon, Guyll, Spoth, Cross, and Hilbert (2003) explored the mediating role of mothers' expectations in the relationship between several background variables and children's future alcohol use. They hypothesized that several variables potentially moderated the effect of maternal expectations on alcohol use. Similarly, Mandel and Johnson (2002) found that the effect of prime (quality vs. money) on product preference was mediated by browsing behavior (in terms of looking time). Furthermore, the effect of browsing behavior on preference was moderated by expertise. Tein, Sandler, and Zautra (2000) investigated the role of psychological distress in mediating the relationship between major and minor stressors and mothers' parenting, enriching their study by examining the role of coping in moderating the relationship between distress and parenting. Donaldson (2001) described a body of research showing that the effects of an intervention program on mental health and employment are carried by several mediators, but that the effects of these mediators on the outcomes are moderated by pretest mental health.

Figure 2, Panel C depicts the path model used to investigate such processes. Again using a method analogous to that used in previous sections, the conditional indirect effect of $X$ on $Y$ is expressed as $f(\hat{\boldsymbol{\theta}} \mid W)=\hat{a}_{1}\left(\hat{b}_{1}+\hat{b}_{3} W\right)$.

Model 4: When the $a$ Path Is Moderated by $W$ and the $b$ Path Is Moderated by $Z$

A simple extension of Models 2 and 3 may be imagined, in which the $a_{1}$ and $b_{1}$ paths of an otherwise simple mediation model are moderated by different variables. For example, Donaldson (2001) described a complex body of research investigating the effects of intervention programs on adolescent drug use. Direct effects were hypothesized to be mediated by programmatic effects on beliefs 
about acceptability, prevalence estimates, and refusal skills, with some $a$ paths moderated by the type of school (public vs. private) and at least one $b$ path moderated by negative intentions to drink. Slater, Hayes, and Ford (2006) report evidence of an indirect effect of sensation seeking on adolescents' perceptions of the risk of alcohol use through attention to news content about accidents, injuries, and crime. But the size of the indirect effect depends on prior bad experiences with alcohol and amount of exposure to news content. In this example of Model 4, the path from sensation seeking to attention to news content was moderated by prior bad experiences, whereas the effect of attention on risk perceptions was moderated by exposure to general news. Combining Models 2 and 3 yields the model in Figure 2, Panel D. The indirect effect of $X$ on $Y$ is moderated by both $W$ and $Z$, with $f(\hat{\boldsymbol{\theta}} \mid W, Z)=\left(\hat{a}_{1}+\hat{a}_{3} W\right)\left(\hat{b}_{1}+\hat{b}_{3} Z\right)$.

\section{Model 5: When the $a$ and $b$ Paths Are Both Moderated by $W$}

A special case of the preceding model exists when both the $a_{1}$ and $b_{1}$ paths of an otherwise simple mediation model are moderated by W. For example, Murray, Bellavia, Rose, and Griffin (2003) investigated the moderating effect of perceived regard on (a) the effect of relationship events on feelings of vulnerability and on (b) the effect of feelings of vulnerability on approach/avoidance responses to romantic partners. Bauer, Preacher, and Gil (2006) examined this kind of conditional indirect effect in the context of multilevel modeling, operationalizing moderation of the $a$ and $b$ paths as cross-level interaction effects. The model in Figure 2, Panel E is the same as the one Baron and Kenny (1986) described when they discussed moderated mediation and is also the model suggested by Muller et al. (2005) to address both mediated moderation and moderated mediation.

This conditional indirect effect is quantified as $f(\hat{\boldsymbol{\theta}} \mid W)=\left(\hat{a}_{1}+\hat{a}_{3} W\right)\left(\hat{b}_{1}+\right.$ $\hat{b}_{2} W$ ) (Muller et al., 2005). As Muller et al. noted, this quantification of the indirect effect may be used to probe the indirect effect at conditional levels of the moderator, and even suggest that the methods examined by MacKinnon et al. (2002) could be extended to provide a formal hypothesis test. In a later section we do just that.

\section{USING BOOTSTRAPPING TO ASSESS MODERATED MEDIATION}

The previous section described how to generate point estimates for conditional indirect effects for five possible moderated mediation models. We now turn to methods that can be used to test hypotheses about these conditional indirect effects. One approach is to estimate the sampling distribution of the conditional indirect effect nonparametrically through bootstrapping and then use information 
from the bootstrap sampling distribution to generate CIs for the conditional indirect effect. No assumptions need be made about the shape of the sampling distribution, and no particular formula for the $S E$ is required. Bootstrapping has been advocated as an alternative to normal-theory tests of mediation (Lockwood \& MacKinnon, 1998; MacKinnon et al., 2004; Preacher \& Hayes, 2004; Shrout $\&$ Bolger, 2002). Because a conditional indirect effect is merely the product of two causal path estimates conditioned on the value of one or more moderators, bootstrapping can be applied just as readily to the assessment of conditional indirect effects as it can to unconditional indirect effects. To illustrate how to bootstrap a conditional indirect effect, we use Model 3 as an example. However, the logic described here applies to bootstrapping conditional indirect effects in any of the models described in the previous section.

In Model 3, the effect of the mediator variable $(M)$ on the outcome variable $(Y)$ is modeled as moderated by $W$, whereas the effect of the independent variable $(X)$ on the mediator variable $(M)$ is not. From Figure 2, Panel $\mathrm{C}$, the mediator model is:

$$
M=a_{0}+a_{1} X+r
$$

and the dependent variable model is:

$$
Y=b_{0}+b_{1} M+b_{2} W+b_{3} M W+c^{\prime} X+r
$$

As discussed previously, the conditional indirect effect of $X$ on $Y$ through $M$ at $W$ is quantified as $f(\hat{\boldsymbol{\theta}} \mid W)=\hat{a}_{1}\left(\hat{b}_{1}+\hat{b}_{3} W\right)$ where $W$ is any value of interest within the range observed in the data. The sampling distribution of $f(\hat{\boldsymbol{\theta}} \mid W)$ is derived through bootstrapping by estimating the coefficients of the mediator and dependent models $k$ times, with each of the $k$ sets of estimates being based on a different random sample of size $N$ taken with replacement from the original sample of $N$ units. The mediator and dependent variable models can be estimated either simultaneously using SEM software or in separate regression models. If the latter, each resample of size $N$ should be used to estimate both models rather than using a separate resample of size $N$ for each model (which would require $2 k$ resamples). In resample $i$, the conditional indirect effect is $f(\hat{\boldsymbol{\theta}} \mid W)_{i}=\hat{a}_{1 i}\left(\hat{b}_{1 i}+\hat{b}_{3 i} W\right)$. Sorting the $k$ values of $f(\hat{\boldsymbol{\theta}} \mid W)_{i}$ low to high, the lower bound of a $100(1-\alpha) \%$ CI for the indirect effect is the $(\alpha / 2) k^{\text {th }}$ value of $f(\hat{\boldsymbol{\theta}} \mid W)_{i}$ in the sorted distribution, and the $(1+(1-\alpha / 2) k)^{\text {th }}$ value functions as the upper bound. The null hypothesis of no conditional indirect effect can be rejected if the CI does not contain 0. As MacKinnon et al. (2004) show in the context of simple mediator models with no moderation, the application of a bias correction to a percentile CI can improve its accuracy (for computational details, see Efron, 1987; Efron \& Tibshirani, 1998; Lunneborg, 2000; Preacher \& Hayes, 2006; Stine, 1989). Later we present and discuss an SPSS macro 
that can be used to obtain bootstrapped confidence intervals (percentile, biascorrected, and bias-corrected and accelerated) for all of the conditional indirect effects discussed in this article.

As useful as bootstrapping is, it is not without limitations. Of minor concern is that bootstrapped intervals require more computing time to obtain than do normal-theory results, particularly in large samples, but with ever-increasing processor speed this limitation is not considered serious. Of more concern, bootstrapping cannot easily be used to establish regions of significance for conditional indirect effects. We now turn to a normal-theory approach that can be used to obtain regions of significance.

\section{USING NORMAL-THEORY STANDARD ERRORS TO ASSESS MODERATED MEDIATION}

The product of coefficients approach discussed earlier in the context of simple mediation can be extended to more complicated models like those in Figure 2. Bollen (1987, 1989) and Sobel (1982) demonstrated the use of the first-order multivariate delta method in determining $S E$ s of a broad class of indirect effects. For example, the first-order approximation to the asymptotic $S E$ of $f(\hat{\boldsymbol{\theta}} \mid X)$ for the special case of Model 1 is:

$$
S E_{f(\hat{\boldsymbol{\theta}} \mid X)} \approx \sqrt{\left(\hat{b}_{1}+\hat{b}_{2} X\right)^{2} s_{\hat{a}_{1}}^{2}+\hat{a}_{1}^{2}\left(s_{\hat{b}_{1}}^{2}+2 s_{\hat{b}_{1}, \hat{b}_{2}} X+s_{\hat{b}_{2}}^{2} X^{2}\right)}
$$

Full derivation details can be seen in the Technical Appendix.

Assuming normality for $f(\hat{\boldsymbol{\theta}} \mid X)$, an asymptotic significance test of the conditional indirect effect may be conducted by dividing $f(\hat{\boldsymbol{\theta}} \mid X)$ by the standard error of $f(\hat{\boldsymbol{\theta}} \mid X)$ :

$$
z \approx \frac{\hat{a}_{1}\left(\hat{b}_{1}+\hat{b}_{2} X\right)}{\sqrt{\left(\hat{b}_{1}+\hat{b}_{2} X\right)^{2} s_{\hat{a}_{1}}^{2}+\hat{a}_{1}^{2}\left(s_{\hat{b}_{1}}^{2}+2 s_{\hat{b}_{1}, \hat{b}_{2}} X+s_{\hat{b}_{2}}^{2} X^{2}\right)}}
$$

and comparing $z$ to a table of standard normal probabilities.

The test in Equation 14 is appropriate only if the total sample size is large ${ }^{4}$ and the researcher has in mind a limited number of key conditional values of $X$. Alternatively, it may be of interest to discover the range of conditional values of

\footnotetext{
${ }^{4}$ Because the normal-theory approach invokes the assumption that the conditional indirect effect is normally distributed across repeated sampling, the test statistic is only asymptotically normal, meaning that the total sample size should be large if the normality assumption is to be satisfied to a reasonable degree. Bootstrapping may be used in samples of virtually any size.
} 
$X$ for which the test in Equation 14 will be significant at a chosen $\alpha$; i.e., it may be of interest to extend the $\mathrm{J}-\mathrm{N}$ technique to models involving both mediation and moderation. In applying the $\mathrm{J}-\mathrm{N}$ technique, one reverses the equation for the $z$-test associated with the conditional indirect effect, setting $z$ equal to the critical value for the desired $\alpha$ and, assuming a two-tailed test is desired, solving the quadratic formula for the two roots $\left\{X_{\text {low }}, X_{\text {high }}\right\}$ :

$$
\left\{X_{\text {low }}, X_{\text {high }}\right\}=\frac{-B \pm \sqrt{B^{2}-4 A C}}{2 A}
$$

These roots correspond to values of the moderator that would separate significant conditional indirect effects from nonsignificant ones. Depending on the application, the range of values enclosed by the two roots will be either the values of $X$ for which $f(\hat{\boldsymbol{\theta}} \mid X)$ is significant, with those values outside the range corresponding to nonsignificant $f(\hat{\boldsymbol{\theta}} \mid X)$, or the reverse. In the case of Model 1 , $f(\hat{\boldsymbol{\theta}} \mid X)=\hat{a}_{1}\left(\hat{b}_{1}+\hat{b}_{2} X\right)$ and the lower and upper bounds will correspond to roots of Equation 15 for which:

$$
\begin{aligned}
& A=\left(-\hat{a}_{1}^{2} \hat{b}_{2}^{2}+\hat{b}_{2}^{2} s_{\hat{a}_{1}}^{2} z^{2}+\hat{a}_{1}^{2} s_{\hat{b}_{2}}^{2} z^{2}\right) \\
& B=2\left(-\hat{a}_{1}^{2} \hat{b}_{1} \hat{b}_{2}+\hat{a}_{1}^{2} s_{\hat{b}_{1}, \hat{b}_{2}} z^{2}+\hat{b}_{1} \hat{b}_{2} s_{\hat{a}_{1}}^{2} z^{2}\right) \\
& C=\left(-\hat{a}_{1}^{2} \hat{b}_{1}^{2}+\hat{b}_{1}^{2} s_{\hat{a}_{1}}^{2} z^{2}+\hat{a}_{1}^{2} s_{\hat{b}_{1}}^{2} z^{2}\right)
\end{aligned}
$$

where $z_{\alpha / 2}$ is the critical value of $z$.

The $S E$ used for significance testing or CI formation may be approximated more closely by using a second-order delta method to approximate the $S E$ (see Technical Appendix). When $f(\hat{\boldsymbol{\theta}} \mid X)=\hat{a}_{1}\left(\hat{b}_{1}+\hat{b}_{2} X\right)$, the $S E$ becomes:

$$
S E_{f(\hat{\boldsymbol{\theta}} \mid X)} \approx \sqrt{\left(\hat{b}_{1}+\hat{b}_{2} X\right)^{2} s_{\hat{a}_{1}}^{2}+\left(\hat{a}_{1}^{2}+s_{\hat{a}_{1}}^{2}\right)\left(s_{\hat{b}_{1}}^{2}+2 s_{\hat{b}_{1}, \hat{b}_{2}} X+s_{\hat{b}_{2}}^{2} X^{2}\right)}
$$

This $S E$ has a form analogous to that of the second-order $S E$ derived for simple mediation (Equation 3 ), in which $\hat{b}_{1}$ is replaced by its conditional counterpart $\left(\hat{b}_{1}+\hat{b}_{2} X\right) .^{5}$ The roots defining the second-order region of significance are slightly more complex, but are derivable by the same methods as the first-order region; that is, by setting $z$ equal to the desired critical value and solving the

\footnotetext{
${ }^{5}$ We provide both first- and second-order $S E$ s because the differences are often negligible, and both are routinely reported in literature on simple mediation. Future research may determine when (or whether) one is more appropriate than the other.
} 
quadratic formula substituting the following for $A, B$, and $C$ :

$$
\begin{aligned}
& A=\left(-\hat{a}_{1}^{2} \hat{b}_{2}^{2}+\hat{b}_{2}^{2} s_{\hat{a}_{1}}^{2} z^{2}+\hat{a}_{1}^{2} s_{\hat{b}_{2}}^{2} z^{2}+s_{\hat{a}_{1}}^{2} s_{\hat{b}_{1}}^{2} z^{2}\right) \\
& B=2\left(-\hat{a}_{1}^{2} \hat{b}_{1} \hat{b}_{2}+\hat{a}_{1}^{2} s_{\hat{b}_{1}, \hat{b}_{2}} z^{2}+\hat{b}_{1} \hat{b}_{2} s_{\hat{a}_{1}}^{2} z^{2}+s_{\hat{b}_{1}, \hat{b}_{2}} s_{\hat{a}_{1}}^{2} z^{2}\right) \\
& C=\left(-\hat{a}_{1}^{2} \hat{b}_{1}^{2}+\hat{b}_{1}^{2} s_{\hat{a}_{1}}^{2} z^{2}+\hat{a}_{1}^{2} s_{\hat{b}_{1}}^{2} z^{2}+s_{\hat{a}_{1}}^{2} s_{\hat{b}_{1}}^{2} z^{2}\right)
\end{aligned}
$$

Conditional indirect effects and their confidence bands may be plotted against values of the moderator in all of the models we discuss. In general, the formula for a confidence band is:

$$
\mathrm{CB}_{1-\alpha}: f(\hat{\boldsymbol{\theta}} \mid W) \pm z_{\alpha / 2} S E .
$$

The explicit function for plotting (e.g.) $95 \%$ confidence bands for Model 3 is:

$$
\begin{aligned}
\mathrm{CB}_{1-\alpha} & : \hat{a}_{1}\left(\hat{b}_{1}+\hat{b}_{3} W\right) \pm 1.96 \\
\times & \sqrt{\left(\hat{b}_{1}+\hat{b}_{3} W\right)^{2} s_{\hat{a}_{1}}^{2}+\left(\hat{a}_{1}^{2}+s_{\hat{a}_{1}}^{2}\right)\left(s_{\hat{b}_{1}}^{2}+2 s_{\hat{b}_{1}, \hat{b}_{3}} W+s_{\hat{b}_{3}}^{2} W^{2}\right)} .
\end{aligned}
$$

Equation 20 illustrates how the magnitude of the conditional indirect effectas well as the width of its confidence interval-depends on the value of the moderator.

Similar methods may be used to derive first- and second-order SEs for all of the models in Figure 2. Similarly, regions of significance and confidence bands may be computed for conditional indirect effects in any of the models we have discussed. In Model 4, such plots can be produced for conditional values of either $W$ or $Z$. In Model 5, the conditional indirect effects and confidence bands take on some very interesting quadratic characteristics. To clarify and organize our results, we summarize the normal-theory results for indirect effects discussed in this article in Table 1. The first column of Table 1 contains labels for each model (Models 0-5, where Model 0 is the simple or unconditional mediation model and Models 1-5 are conditional models). The second column contains the point estimate of the indirect effect of interest. Finally, the third column contains the variances to be used in hypothesis tests in the construction of confidence intervals, regions of significance, and confidence bands. The paths referenced in each equation refer to those labeled in Figures 1 and 2. Table 2 contains the $\mathrm{A}, \mathrm{B}$, and $\mathrm{C}$ values for use with the quadratic formula for computing regions of significance for Models 1 to 3 .

The $S E$ of a conditional indirect effect may be used to assess mediation at any chosen value of the moderator(s) so long as the value lies within the observed 
TABLE 1

Indirect Effect Point Estimates and Second-Order Variances

\begin{tabular}{|c|c|c|}
\hline Model & Point Estimate & Second-Order Variance \\
\hline 0 & $\hat{a}_{1} \hat{b}_{1}$ & $\hat{b}_{1}^{2} s_{\hat{a}_{1}}^{2}+\hat{a}_{1}^{2} s_{\hat{b}_{1}}^{2}+s_{\hat{a}_{1}}^{2} s_{\hat{b}_{1}}^{2}$ \\
\hline 1 & $\hat{a}_{1}\left(\hat{b}_{1}+\hat{b}_{2} X\right)$ & $\left(\hat{b}_{1}+\hat{b}_{2} X\right)^{2} s_{\hat{a}_{1}}^{2}+\left(\hat{a}_{1}^{2}+\underline{s}_{\hat{a}_{1}}^{2}\right)\left(s_{\hat{b}_{1}}^{2}+2 s_{\hat{b}_{1}, \hat{b}_{2}} X+s_{\hat{b}_{2}}^{2} X^{2}\right)$ \\
\hline 2 & $\left(\hat{a}_{1}+\hat{a}_{3} W\right) \hat{b}_{1}$ & 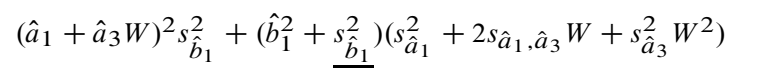 \\
\hline 3 & $\hat{a}_{1}\left(\hat{b}_{1}+\hat{b}_{3} W\right)$ & $\left(\hat{b}_{1}+\hat{b}_{3} W\right)^{2} s_{\hat{a}_{1}}^{2}+\left(\hat{a}_{1}^{2}+\underline{s_{\hat{a}_{1}}^{2}}\right)\left(s_{\hat{b}_{1}}^{2}+2 s_{\hat{b}_{1}, \hat{b}_{3}} W+s_{\hat{b}_{3}}^{2} W^{2}\right)$ \\
\hline 4 & $\begin{array}{l}\left(\hat{a}_{1}+\hat{a}_{3} W\right) \\
\quad \times\left(\hat{b}_{1}+\hat{b}_{3} Z\right)\end{array}$ & $\begin{array}{l}\left(\hat{a}_{1}+\hat{a}_{3} W\right)^{2}\left(s_{\hat{b}_{1}}^{2}+2 s_{\hat{b}_{1}, \hat{b}_{3}} Z+s_{\hat{b}_{3}}^{2} Z^{2}\right) \\
\quad+\left(\hat{b}_{1}+\hat{b}_{3} Z\right)^{2}\left(s_{\hat{a}_{1}}^{2}+2 s_{\hat{a}_{1}, \hat{a}_{3}} W+s_{\left.\hat{a}_{3} W^{2}\right)}^{2}\right. \\
\quad+\left(s_{\hat{b}_{1}}^{2}+2 s_{\hat{b}_{1}, \hat{b}_{3}} Z+s_{\hat{b}_{3}}^{2} Z^{2}\right)\left(s_{\hat{a}_{1}}^{2}+2 s_{\hat{a}_{1}, \hat{a}_{3}} W+s_{\hat{a}_{3}}^{2} W^{2}\right)\end{array}$ \\
\hline 5 & $\begin{array}{l}\left(\hat{a}_{1}+\hat{a}_{3} W\right) \\
\quad \times\left(\hat{b}_{1}+\hat{b}_{2} W\right)\end{array}$ & $\begin{array}{l}\left(\hat{b}_{1}+\hat{b}_{2} W\right)^{2}\left(s_{\hat{a}_{1}}^{2}+2 s_{\hat{a}_{1}, \hat{a}_{3}} W+s_{\hat{a}_{3}}^{2} W^{2}\right) \\
\quad+\left(\hat{a}_{1}+\hat{a}_{3} W\right)^{2}\left(s_{\hat{b}_{1}}^{2}+2 s_{\hat{b}_{1}, \hat{b}_{2}} W+s_{\hat{b}_{2}}^{2} W^{2}\right) \\
\quad+\left(s_{\hat{a}_{1}}^{2}+2 s_{\hat{a}_{1}, \hat{a}_{3}} W+s_{\hat{a}_{3}}^{2} W^{2}\right)\left(s_{\hat{b}_{1}}^{2}+2 s_{\hat{b}_{1}, \hat{b}_{2}} W+s_{\hat{b}_{2}}^{2} W^{2}\right)\end{array}$ \\
\hline
\end{tabular}

Note. The part of each second-order variance that may be omitted to yield the first-order variance has been underlined in each formula. In all cases, the square root of the variance is the $S E$ that can be used to form a critical ratio.

range. Morgan-Lopez and MacKinnon (2006) apply this method (using the firstorder $S E$ approximation) in a study of tobacco use among Mexican-American youth. This $S E$ may also be used to solve for values of $W$ (or $X$ or $Z$ ) for which the indirect effect of $X$ on $Y$ is significant by applying the $\mathrm{J}-\mathrm{N}$ technique and using Equation 15. A more precise region of significance may be obtained by using the second-order approximation instead of the first-order $S E$ to solve for the boundaries of the region of significance. Because Model 4 involves two moderators, obtaining regions of significance is more difficult than for the other models we have discussed. We suggest choosing conditional values of one moderator (either $W$ or $Z$ ) and obtaining regions of significance for the other moderator at those conditional values.

\section{SIMULATION}

We performed a simulation study to examine the Type I error rates and power of the methods examined here under a variety of conditions to provide some 
TABLE 2

A, B, and C Components of the Quadratic Formula for Obtaining Regions of Significance

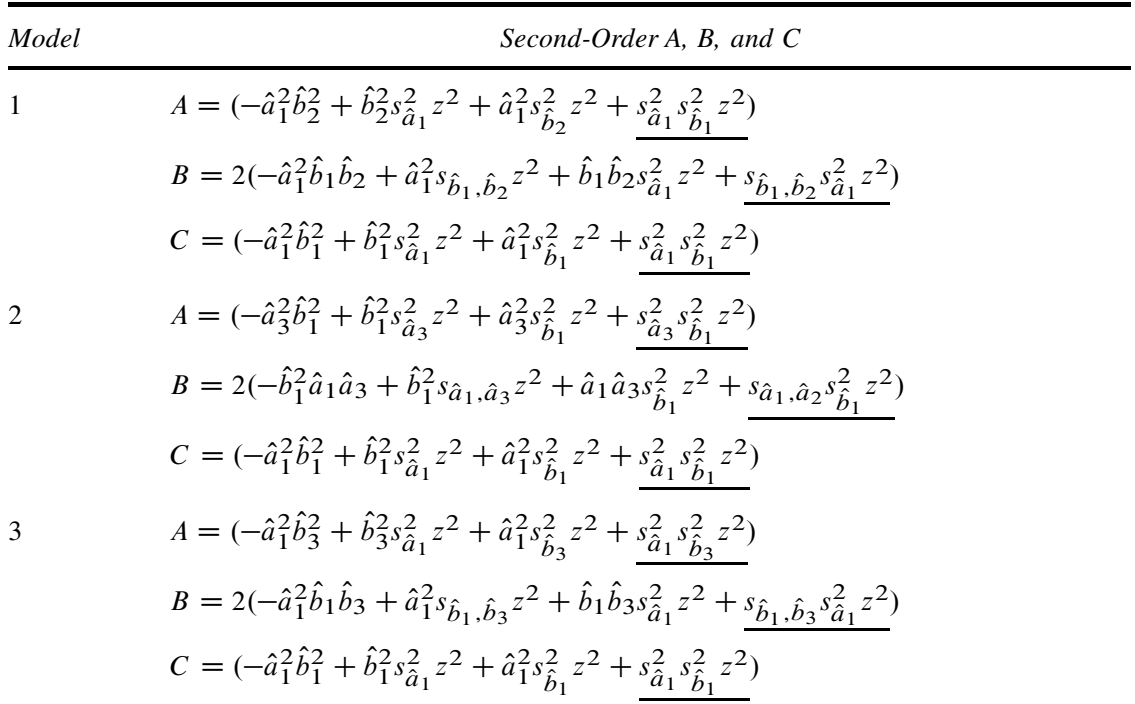

4, 5 Solutions for $A, B$, and $C$ are not provided for Models 4 or 5 because the general expressions are too long to print here. Regions of significance may be obtained by substituting estimated values for all relevant regression coefficients and estimated asymptotic variances and covariances into the $z$ formula, fixing $z$ at the desired critical values, and using general equation-solving software (such as Maple, MATLAB, or Mathematica) to solve for $W$ in the case of Model 4, or for $W$ or $Z$ in the case of Model 5 (fixing $W$ to obtain regions for $Z$ or vice versa). Mathematica code for obtaining regions of significance for Models $1-5$ has been posted at http://www.quantpsy.org/.

Note. The part of each second-order $A, B$, and $C$ that may be omitted to yield the first-order solution has been underlined in each formula.

guidance on appropriate sample sizes. To simplify the study, in all models $a_{1}=$ $a_{3}=b_{1}=b_{2}=b_{3}$ and $c^{\prime}=0$ in the population. Also, all conditional values of the moderator(s) are +1.0 , and all variables are drawn from normally distributed populations with zero means and unit variances. Results are reported in Tables 3-7. Where $a=b=0$, cell values represent empirical Type I error rates. All other cell values represent empirical power.

All cells in Tables 3 to 7 represent proportions of 1,000 trials found significant at $\alpha=.05$ in two-tailed $z$-tests (bootstrapping cells used 1,000 resamples). In every case, rejection rates for $z$-tests using second-order delta method variances led to equal or slightly lower rejection rates than did tests using first-order variances. These results should be interpreted in light of previous studies of bias 
TABLE 3

Type I Error Rates and Empirical Power for Model 1

\begin{tabular}{|c|c|c|c|c|c|c|}
\hline \multirow[b]{2}{*}{ Test } & \multirow{2}{*}{$\begin{array}{l}\text { Regression } \\
\text { Coefficients }\end{array}$} & \multicolumn{5}{|c|}{ Sample Size } \\
\hline & & 50 & 100 & 200 & 500 & 1000 \\
\hline first & .00 & .000 & .000 & .000 & .000 & .000 \\
\hline first & .14 & .001 & .005 & .046 & .316 & .792 \\
\hline first & .39 & .210 & .613 & .975 & 1.000 & 1.000 \\
\hline first & .59 & .710 & .978 & 1.000 & 1.000 & 1.000 \\
\hline second & .00 & .000 & .000 & .000 & .000 & .000 \\
\hline second & .14 & .001 & .003 & .040 & .282 & .769 \\
\hline second & .39 & .187 & .588 & .972 & 1.000 & 1.000 \\
\hline second & .59 & .689 & .976 & 1.000 & 1.000 & 1.000 \\
\hline boot & .00 & .000 & .002 & .001 & .003 & .000 \\
\hline boot & .14 & .015 & .031 & .140 & .514 & .852 \\
\hline boot & .39 & .322 & .720 & .977 & 1.000 & 1.000 \\
\hline boot & .59 & .769 & .983 & 1.000 & 1.000 & 1.000 \\
\hline $\mathrm{bc}$ & .00 & .003 & .007 & .005 & .007 & .005 \\
\hline $\mathrm{bc}$ & .14 & .030 & .065 & .223 & .623 & .891 \\
\hline $\mathrm{bc}$ & .39 & .414 & .786 & .982 & 1.000 & 1.000 \\
\hline bc & .59 & .818 & .987 & 1.000 & 1.000 & 1.000 \\
\hline bca & .00 & .003 & .007 & .005 & .007 & .005 \\
\hline bca & .14 & .030 & .065 & .223 & .623 & .891 \\
\hline bca & .39 & .414 & .785 & .982 & 1.000 & 1.000 \\
\hline bca & .59 & .818 & .987 & 1.000 & 1.000 & 1.000 \\
\hline
\end{tabular}

Note. In this and subsequent tables, first refers to tests using first-order standard errors, second to second-order standard errors, boot to rejection rates using percentile-based bootstrapped confidence intervals, $b c$ to bias-corrected limits, and $b c a$ to bias-corrected and accelerated limits.

in standard errors of indirect effects. MacKinnon (1992, Table 1) found that second-order $S E$ s for simple mediation effects were less biased than first-order $S E$ s, but MacKinnon et al. (1995, Table 4) found the opposite. Here, bootstrapping showed higher power and closer-to-accurate (although still poor) Type I error rates than delta method results; bias-corrected and $\mathrm{BCa}$ results showed particularly high power. ${ }^{6}$ Of interest, statistical power for detecting conditional

\footnotetext{
${ }^{6}$ The conditions we investigated in this simulation do not exhaust the possibilities. A full-scale simulation study investigating other conditions would be helpful. Future research should investigate rejection rates when relevant path coefficients are unequal, and for conditional values of the moderator(s) other than +1.0 .
} 
TABLE 4

Type I Error Rates and Empirical Power for Model 2

\begin{tabular}{|c|c|c|c|c|c|c|}
\hline \multirow[b]{2}{*}{ Test } & \multirow{2}{*}{$\begin{array}{l}\text { Regression } \\
\text { Coefficients }\end{array}$} & \multicolumn{5}{|c|}{ Sample Size } \\
\hline & & 50 & 100 & 200 & 500 & 1000 \\
\hline first & .00 & .000 & .000 & .000 & .000 & .001 \\
\hline first & .14 & .006 & .032 & .200 & .798 & .988 \\
\hline first & .39 & .521 & .942 & 1.000 & 1.000 & 1.000 \\
\hline first & .59 & .950 & .999 & 1.000 & 1.000 & 1.000 \\
\hline second & .00 & .000 & .000 & .000 & .000 & .001 \\
\hline second & .14 & .005 & .025 & .170 & .784 & .987 \\
\hline second & .39 & .488 & .937 & 1.000 & 1.000 & 1.000 \\
\hline second & .59 & .943 & .999 & 1.000 & 1.000 & 1.000 \\
\hline boot & .00 & .002 & .000 & .001 & .001 & .002 \\
\hline boot & .14 & .024 & .099 & .348 & .880 & .993 \\
\hline boot & .39 & .630 & .950 & 1.000 & 1.000 & 1.000 \\
\hline boot & .59 & .964 & 1.000 & 1.000 & 1.000 & 1.000 \\
\hline $\mathrm{bc}$ & .00 & .005 & .003 & .002 & .004 & .009 \\
\hline $\mathrm{bc}$ & .14 & .049 & .175 & .460 & .912 & .993 \\
\hline $\mathrm{bc}$ & .39 & .706 & .962 & 1.000 & 1.000 & 1.000 \\
\hline $\mathrm{bc}$ & .59 & .970 & 1.000 & 1.000 & 1.000 & 1.000 \\
\hline bca & .00 & .004 & .003 & .002 & .004 & .009 \\
\hline bca & .14 & .050 & .174 & .461 & .912 & .993 \\
\hline bca & .39 & .705 & .962 & 1.000 & 1.000 & 1.000 \\
\hline bca & .59 & .970 & 1.000 & 1.000 & 1.000 & 1.000 \\
\hline
\end{tabular}

indirect effects was noticeably larger for Models 4 and 5 than for Models 2 and 3, which in turn demonstrated higher power than Model 1. This trend was evident for all five of the methods we compared.

\section{COMPUTER METHODS FOR PERFORMING MODERATED MEDIATION ANALYSES}

The calculations necessary for producing the first- and second-order variances of conditional indirect effects and for deriving regions of significance are laborious at best, and the potential for error in hand computation is high. Moreover, bootstrapping simply cannot be accomplished without computer programs. In this section, we discuss two means of implementing the methods we discuss here that avoid or substantially reduce hand computation and the potential for error. 
TABLE 5

Type I Error Rates and Empirical Power for Model 3

\begin{tabular}{|c|c|c|c|c|c|c|}
\hline \multirow[b]{2}{*}{ Test } & \multirow{2}{*}{$\begin{array}{l}\text { Regression } \\
\text { Coefficients }\end{array}$} & \multicolumn{5}{|c|}{ Sample Size } \\
\hline & & 50 & 100 & 200 & 500 & 1000 \\
\hline first & .00 & .001 & .000 & .000 & .000 & .000 \\
\hline first & .14 & .006 & .041 & .196 & .794 & .985 \\
\hline first & .39 & .563 & .945 & 1.000 & 1.000 & 1.000 \\
\hline first & .59 & .957 & 1.000 & 1.000 & 1.000 & 1.000 \\
\hline second & .00 & .001 & .000 & .000 & .000 & .000 \\
\hline second & .14 & .004 & .029 & .175 & .774 & .984 \\
\hline second & .39 & .529 & .935 & 1.000 & 1.000 & 1.000 \\
\hline second & .59 & .953 & 1.000 & 1.000 & 1.000 & 1.000 \\
\hline boot & .00 & .001 & .000 & .000 & .001 & .002 \\
\hline boot & .14 & .029 & .112 & .341 & .861 & .990 \\
\hline boot & .39 & .659 & .949 & 1.000 & 1.000 & 1.000 \\
\hline boot & .59 & .969 & .999 & 1.000 & 1.000 & 1.000 \\
\hline $\mathrm{bc}$ & .00 & .003 & .006 & .005 & .005 & .004 \\
\hline $\mathrm{bc}$ & .14 & .067 & .182 & .456 & .894 & .991 \\
\hline $\mathrm{bc}$ & .39 & .737 & .963 & 1.000 & 1.000 & 1.000 \\
\hline bc & .59 & .972 & .999 & 1.000 & 1.000 & 1.000 \\
\hline bca & .00 & .003 & .006 & .005 & .005 & .004 \\
\hline bca & .14 & .068 & .182 & .456 & .894 & .991 \\
\hline bca & .39 & .737 & .963 & 1.000 & 1.000 & 1.000 \\
\hline bca & .59 & .973 & .999 & 1.000 & 1.000 & 1.000 \\
\hline
\end{tabular}

\section{Moderated Mediation SPSS Macro}

To facilitate the computations described in this article and to make these methods available to researchers without access to software that can bootstrap "out of the can" or who have limited experience with SEM software, we have written an SPSS macro that implements all the methods described in this article. A macro is a sequence of commands that define new functions the user can control to conduct custom analyses. The macro, once executed, creates a new command in SPSS called MODMED. Using the MODMED command, the user provides information about which variables in the model to be estimated function as the independent variable, the mediator, the outcome, and the moderator in the desired analysis. Depending on how these variables are listed in the command line, SPSS estimates the coefficients for one of the five moderated mediation models described in this paper and depicted graphically in Figure 2. For instance, suppose the independent variable $(X)$ is disposition, the mediator $(M)$ variable is shyness, the dependent variable $(Y)$ is happy, and there are two additional 
PREACHER, RUCKER, HAYES

TABLE 6

Type I Error Rates and Empirical Power for Model 4

\begin{tabular}{|c|c|c|c|c|c|c|}
\hline \multirow[b]{2}{*}{ Test } & \multirow{2}{*}{$\begin{array}{l}\text { Regression } \\
\text { Coefficients }\end{array}$} & \multicolumn{5}{|c|}{ Sample Size } \\
\hline & & 50 & 100 & 200 & 500 & 1000 \\
\hline first & .00 & .000 & .000 & .000 & .000 & .000 \\
\hline first & .14 & .017 & .069 & .393 & .965 & 1.000 \\
\hline first & .39 & .751 & .996 & 1.000 & 1.000 & 1.000 \\
\hline first & .59 & .988 & 1.000 & 1.000 & 1.000 & 1.000 \\
\hline second & .00 & .000 & .000 & .000 & .000 & .000 \\
\hline second & .14 & .012 & .060 & .364 & .958 & 1.000 \\
\hline second & .39 & .727 & .996 & 1.000 & 1.000 & 1.000 \\
\hline second & .59 & .987 & 1.000 & 1.000 & 1.000 & 1.000 \\
\hline boot & .00 & .001 & .002 & .002 & .000 & .005 \\
\hline boot & .14 & .049 & .178 & .586 & .980 & 1.000 \\
\hline boot & .39 & .778 & .997 & 1.000 & 1.000 & 1.000 \\
\hline boot & .59 & .988 & 1.000 & 1.000 & 1.000 & 1.000 \\
\hline $\mathrm{bc}$ & .00 & .005 & .004 & .007 & .003 & .008 \\
\hline $\mathrm{bc}$ & .14 & .086 & .271 & .696 & .988 & 1.000 \\
\hline $\mathrm{bc}$ & .39 & .853 & .998 & 1.000 & 1.000 & 1.000 \\
\hline $\mathrm{bc}$ & .59 & .991 & 1.000 & 1.000 & 1.000 & 1.000 \\
\hline bca & .00 & .005 & .004 & .007 & .003 & .008 \\
\hline bca & .14 & .086 & .273 & .695 & .988 & 1.000 \\
\hline bca & .39 & .852 & .998 & 1.000 & 1.000 & 1.000 \\
\hline bca & .59 & .991 & 1.000 & 1.000 & 1.000 & 1.000 \\
\hline
\end{tabular}

potential moderator variables: activity $(W)$ and $i q(Z)$. Models 1 through 5 would be estimated with the following commands:

Model 1

modmed $\mathrm{dv}=$ happy $/$ med $=$ shyness $/ \mathrm{dvmodel}=$ shyness $\operatorname{disposition} / \mathrm{mmodel}$

$$
=\text { disposition. }
$$

Model 2

$$
\begin{aligned}
\operatorname{modmed} \mathrm{dv} & =\text { happy } / \text { med }=\text { shyness } / \mathrm{d} \text { model }=\text { shyness } / \text { mmodel } \\
& =\text { disposition activity. }
\end{aligned}
$$


TABLE 7

Type I Error Rates and Empirical Power for Model 5

\begin{tabular}{|c|c|c|c|c|c|c|}
\hline \multirow[b]{2}{*}{ Test } & \multirow{2}{*}{$\begin{array}{l}\text { Regression } \\
\text { Coefficients }\end{array}$} & \multicolumn{5}{|c|}{ Sample Size } \\
\hline & & 50 & 100 & 200 & 500 & 1000 \\
\hline first & .00 & .000 & .002 & .000 & .000 & .000 \\
\hline first & .14 & .016 & .074 & .363 & .952 & 1.000 \\
\hline first & .39 & .747 & .989 & 1.000 & 1.000 & 1.000 \\
\hline first & .59 & .989 & 1.000 & 1.000 & 1.000 & 1.000 \\
\hline second & .00 & .000 & .001 & .000 & .000 & .000 \\
\hline second & .14 & .016 & .062 & .328 & .939 & 1.000 \\
\hline second & .39 & .730 & .989 & 1.000 & 1.000 & 1.000 \\
\hline second & .59 & .986 & 1.000 & 1.000 & 1.000 & 1.000 \\
\hline boot & .00 & .005 & .004 & .003 & .000 & .002 \\
\hline boot & .14 & .051 & .185 & .559 & .975 & 1.000 \\
\hline boot & .39 & .784 & .993 & 1.000 & 1.000 & 1.000 \\
\hline boot & .59 & .981 & 1.000 & 1.000 & 1.000 & 1.000 \\
\hline $\mathrm{bc}$ & .00 & .011 & .010 & .005 & .003 & .006 \\
\hline bc & .14 & .085 & .297 & .656 & .991 & 1.000 \\
\hline $\mathrm{bc}$ & .39 & .858 & .994 & 1.000 & 1.000 & 1.000 \\
\hline bc & .59 & .988 & 1.000 & 1.000 & 1.000 & 1.000 \\
\hline bca & .00 & .011 & .009 & .005 & .003 & .006 \\
\hline bca & .14 & .088 & .295 & .655 & .991 & 1.000 \\
\hline bca & .39 & .857 & .994 & 1.000 & 1.000 & 1.000 \\
\hline bca & .59 & .991 & 1.000 & 1.000 & 1.000 & 1.000 \\
\hline
\end{tabular}

\section{Model 3}

modmed $\mathrm{dv}=$ happy $/$ med $=$ shyness $/ \mathrm{dvmodel}=$ shyness activity $/$ mmodel

$$
=\text { disposition. }
$$

Model 4

$$
\begin{aligned}
\operatorname{modmed} \mathrm{dv} & =\text { happy } / \text { med }=\text { shyness } / \text { dvmodel }=\text { shyness } \mathrm{iq} / \text { mmodel } \\
& =\text { disposition activity. }
\end{aligned}
$$

\section{Model 5}

modmed $\mathrm{dv}=$ happy $/$ med $=$ shyness $/ \mathrm{dvmodel}=$ shyness activity $/$ mmodel

$$
=\text { disposition activity. }
$$


If no options are specified, the macro defaults to printing the estimates of the model and the conditional indirect effects and hypothesis tests conditioned on the moderators being set to the sample mean and $\pm 1 S D$ as well as conditional indirect effects at values of the moderator in various increments within the range of the data. If they exist, the macro also produces the conditional indirect effect at the value of the moderator(s) for which the effect is just statistically significant (at $\alpha=.05$ ) using the $\mathrm{J}-\mathrm{N}$ technique. Hypothesis tests use the second-order $S E$ s by default. An option allows the user to specify first-order $S E$ s if desired. Another option allows the user to specify precise values of the moderator(s) for which conditional indirect effects and hypothesis tests are produced in the output. A similar version of the macro (MODMEDC) allows for the inclusion of covariates in the mediator and outcome variable models.

Because of the computational time required, the macro does not provide bootstrap estimates of confidence intervals by default, but we encourage the user to request bootstrap estimates routinely. The user can control whether bootstrapping is used and, if so, the number of bootstrap samples taken, in increments of 1,000, as well as whether the macro generates confidence intervals using the percentile, bias corrected, and bias corrected and accelerated methods. The macro can be obtained by contacting any of the authors or from http://www.quantpsy.org/ where detailed documentation and a worked example are provided. We further discuss the use of the macro in the next section, Example Application.

Example application. To illustrate how Model 3 might be specified and probed using our macro, we made use of data collected as part of the Michigan Study of Adolescent Life Transitions (MSALT; Eccles, 1988). This longitudinal study followed adolescents in 12 Michigan school districts from elementary to junior high school and beyond. The MSALT data emphasize the measurement of classroom and school environment variables and teacher and parent perceptions in an attempt to identify determinants of future student achievement-relevant beliefs, motives, values, and behaviors. Our model is loosely based on hypotheses examined by Madon, Jussim, and Eccles (1997). Madon et al. (p. 794) described a conceptual model in which several background variables affect teacher perceptions and misperceptions of student performance, talent, and effort, which may in turn predict future academic performance (i.e., self-fulfilling prophecies) after controlling for relevant background predictors. The effect of teacher perceptions on student performance was hypothesized to be moderated by (among other things) students' academic self-concept and previous achievement, as well as by teacher over- and underestimates of students along various dimensions. Madon et al. did not test the full conceptual model. We show how this task might be approached within our proposed framework.

We chose to model the indirect effect of student intrinsic interest in math (INTRINT; sixth grade) on math performance at the end of the eighth grade 
(MATH8) through teacher perceptions of talent (PERCTAL; seventh grade). This indirect effect, in turn, was hypothesized to be moderated by math selfconcept (SCMATH; eighth grade) as assessed using our Model 3. That is, the indirect effect of INTRINT on MATH8 was hypothesized to be moderated by SCMATH because SCMATH moderates the effect of PERCTAL on MATH8. ${ }^{7}$ Intrinsic interest was assessed by combining two self-report items: "In general, I find working on math assignments..." (1 [very boring], 7 [very interesting] $)$ and "How much do you like doing math?" (1 [a little], 7 [a lot]). Math self-concept was assessed by combining two items: "How good at math are you?" (1 [not at all good], 7 [very good]) and "If you were to rank all the students in your math class from the worst to the best in math, where would you put yourself?" (1 [the worst], 7 [the best]). Teacher perceptions were assessed with the item "How much natural mathematical talent does this student have?" (1 [very little math talent], 7 [a lot of math talent]). The sample size was 609.

With no moderator in the model, the indirect effect was found to be significant using the SPSS macro discussed in Preacher and Hayes (2004) to obtain both a bootstrapped confidence interval (95\% CI: $\{0.1812,0.3320\}$ with 5,000 resamples) and the product of coefficients approach using a second-order $S E$ estimate (Sobel $z=6.1789, p<.001$ ). The signs of the path coefficients and the indirect effect were consistent with the interpretation that intrinsic interest increases teacher perceptions of math ability, which in turn influences math performance.

Using the SPSS macro introduced here and described earlier, we then estimated the coefficients of a moderated mediation model in which this indirect effect was presumed to be moderated by math self-concept. Note that a significant unconditional indirect effect does not constitute a prerequisite for examining conditional indirect effects. The command syntax was

$$
\begin{aligned}
\text { modmed } \mathrm{dv} & =\text { math } 8 / \text { med }=\text { perctal } / \text { dvmodel }=\text { perctal scmath } / \text { mmodel } \\
& =\text { intrint. }
\end{aligned}
$$

Relevant components of the output of the macro are displayed in Table 8. The statistically significant interaction between SCMATH and PERCTAL in the model for MATH8 implies that the indirect effect of intrinsic interest on math performance through teacher perceptions is moderated by SCMATH. The sign of the interaction is consistent with the interpretation that the indirect effect is larger for students with higher math self-concepts. Given the interaction, it makes sense to probe the indirect effect by estimating conditional indirect effects at

\footnotetext{
${ }^{7}$ The reader should be cautious in interpreting these effects. A more rigorous analysis would include several control variables and would consider the hierarchical nature of the data set (i.e., students nested within classrooms), factors we ignore here for the sake of pedagogical simplicity.
} 
TABLE 8

Regression Results for Applied Example

\begin{tabular}{|c|c|c|c|c|}
\hline \multirow[b]{2}{*}{ Predictor } & \multicolumn{4}{|c|}{ Mediator Variable Model } \\
\hline & $B$ & $S E$ & $t$ & $p$ \\
\hline Constant & 3.597 & .154 & 23.294 & .000 \\
\hline \multirow[t]{2}{*}{ INTRINT } & .211 & .030 & 6.995 & .000 \\
\hline & \multicolumn{4}{|c|}{ Dependent Variable Model } \\
\hline Predictor & $B$ & $S E$ & $z$ & $p$ \\
\hline Constant & 3.020 & 1.327 & 2.276 & .023 \\
\hline INTRINT & .021 & .066 & .324 & .746 \\
\hline PERCTAL & .131 & .313 & .418 & .676 \\
\hline SCMATH & .550 & .276 & 1.990 & .047 \\
\hline \multirow{3}{*}{$\begin{array}{c}\text { PERCTAL } \times \\
\text { SCMATH }\end{array}$} & .142 & .060 & 2.351 & .019 \\
\hline & & & & \\
\hline & \multicolumn{4}{|c|}{ Conditional Effects at $S C M A T H=$ mean and $\pm 1 S D$} \\
\hline SCMATH $(W)$ & $\hat{a}_{1}\left(\hat{b}_{1}+\hat{b}_{3} W\right)$ & $S E$ & $z$ & $p$ \\
\hline 3.6044 & .135 & .032 & 4.222 & .000 \\
\hline 4.9245 & .175 & .031 & 5.630 & .000 \\
\hline \multirow[t]{2}{*}{6.2446} & .214 & .039 & 5.460 & .000 \\
\hline & \multicolumn{4}{|c|}{ Conditional Effect at Range of Values of SCMATH } \\
\hline SCMATH $(W)$ & $\hat{a}_{1}\left(\hat{b}_{1}+\hat{b}_{3} W\right)$ & $S E$ & $z$ & $p$ \\
\hline 1.0000 & .057 & .055 & 1.044 & .296 \\
\hline 1.3000 & .066 & .052 & 1.286 & .199 \\
\hline 1.6000 & .075 & .048 & 1.557 & .120 \\
\hline 1.9000 & .084 & .045 & 1.861 & .063 \\
\hline 1.9902 & .087 & .044 & 1.960 & .050 \\
\hline 2.2000 & .093 & .042 & 2.202 & .028 \\
\hline 2.5000 & .102 & .040 & 2.581 & .010 \\
\hline 2.8000 & .111 & .037 & 2.996 & .003 \\
\hline$\ldots$ & & & & \\
\hline
\end{tabular}

Note. Table 8 represents a truncated version of the output obtainable from our macro.

values of the moderator. By default, the output displays the conditional indirect effect at three values of the moderator variable: the mean (4.9245), one standard deviation above the mean (6.2446) and one standard deviation below (3.6044), along with normal-theory tests of the hypothesis that the conditional indirect 
effect equals zero. Normal-theory tests are printed by default because they are computationally faster to generate than bootstrap results in the relatively slow SPSS matrix language, making it feasible to produce the large amount of output the macro can produce very quickly. As we discussed, these should be interpreted with some caution and, ideally, verified with bootstrapping. As can be seen, all three of these conditional indirect effects were positive and different from zero, but these tests assume normality of the sampling distribution of the conditional indirect effect (a reasonable assumption in a sample of this size).

To obtain bootstrap confidence intervals for these conditional indirect effects, "/boot $=5000 "$ was added as a command line option (yielding 5,000 bootstrap samples), along with the subcommand "/dvmodv $=w$ " where $w$ was the value of the moderator for which the conditional indirect effect was desired. Setting $w$ to 4.9245 yielded a bootstrap $95 \%$ BCa CI of $\{0.1210,0.2378\}$ (not listed in Table 8). Because this interval does not contain 0 , the conditional indirect effect at SCMATH $=4.9245$ is significantly different from 0 at $\alpha=.05$. Repeating this procedure for $w=3.6044$ and 6.2446 yielded 95\% BCa CIs of $\{0.0756$, $0.2062\}$ and $\{0.1461,0.3051\}$, respectively. Thus, bootstrapping corroborates the results of the normal-theory tests.

By default, the SPSS macro also displays the conditional indirect effect at numerous values of the moderator within the range of the data along with normal theory hypothesis tests and the value of the moderator for which the conditional indirect effect is just statistically significant at $\alpha=.05$ using the JohnsonNeyman technique. As can be seen in Table 8, the indirect effect was found to be significant at $\alpha=.05$ for any value of SCMATH above 1.99 on this 7-point scale.

The conditional indirect effect of interest can be plotted along with a corresponding confidence band. In Figure 3 , the conditional indirect effect of INTRINT on MATH8 via PERCTAL, $\hat{a}_{1}\left(\hat{b}_{1}+\hat{b}_{3} W\right)=.028+.030$ (SCMATH), is plotted with an accompanying 95\% confidence band based on the secondorder variance. According to the plot, it is easy to see that the indirect effect is significantly different from 0 for any value of SCMATH greater than 1.99. One could generate a similar figure for a bootstrap confidence band by repeatedly requesting a bootstrap CI for increasing values of the moderator and then plotting the upper and lower bounds of each CI as a function of the moderator. Although this method can be labor-intensive, it is recommended when the total sample size is small.

\section{Moderator Centering Approach}

The second computer approach, discussed by Muller et al. (2005) and Tein et al. (2004), is an extension of an approach to probing interactions described by Aiken and West (1991), Darlington (1990), and Judd and McClelland (1989). This 


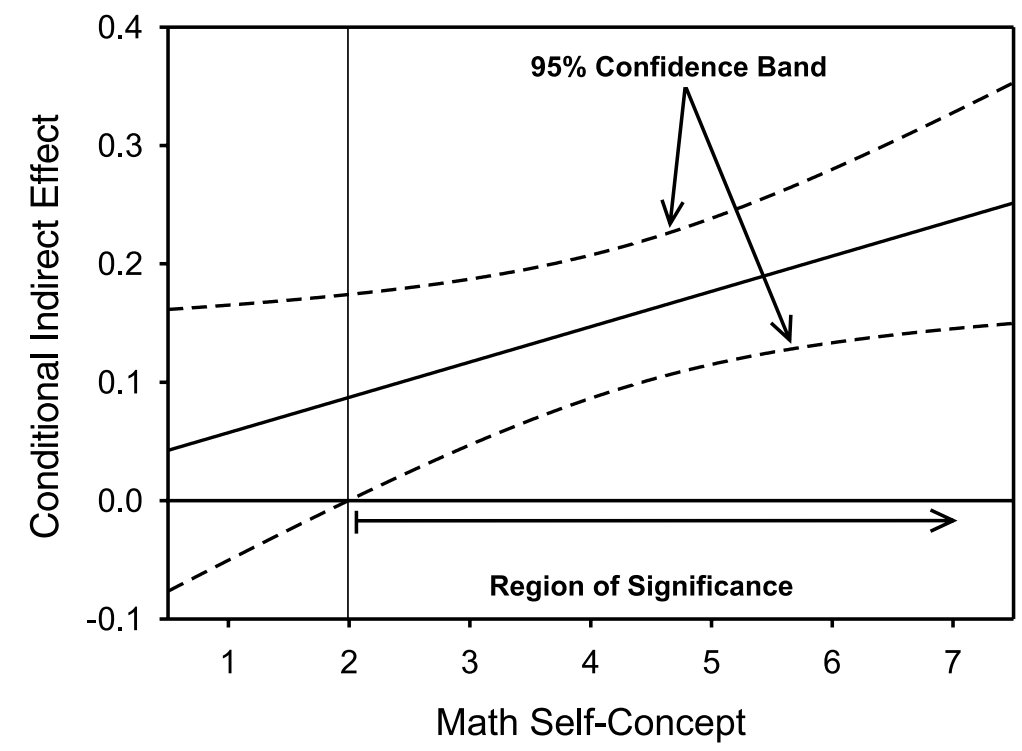

FIGURE 3 A plot of the indirect effect of intrinsic interest on eighth-grade math grades versus the moderator (math self-concept), with confidence bands. The horizontal line denotes an indirect effect of zero. The vertical line represents the boundary of the region of significance.

moderator centering approach involves centering the moderator(s) at conditional values of interest, estimating the model parameters, and interpreting the $X \rightarrow M$ and $M \rightarrow Y$ direct effects as simple slopes. This approach enables simpler tests of indirect effects (e.g., using the $S E$ in Equation 3) to be used in lieu of any of the more complicated tests discussed in the contexts of Models 1 to 5, that do not presume the mediator to be centered at interesting values. Consider, for instance, the conditional indirect effect of early student intrinsic interest in math (INTRINT) on later math performance (MATH8) through teacher perceptions of talent (PERCTAL), conditioned on math self-concept $(\mathrm{SCMATH})=4$, using Model 3. Define $\mathrm{SCMATH}^{\prime}$ as SCMATH-4. Then estimate the two models:

$$
\begin{aligned}
\text { PERCTAL }= & a_{0}+a_{1} \text { INTRINT }+r \\
\text { MATH } 8= & b_{0}+b_{1} \text { PERCTAL }+b_{2} \text { SCMATH }^{\prime} \\
& +b_{3} \text { PERCTAL } \times \text { SCMATH }{ }^{\prime}+c^{\prime} \text { INTRINT }+r^{\text {PERT }}
\end{aligned}
$$

In these models, $a_{1}$ is the effect of early intrinsic interest on teacher perceptions, $b_{1}$ is the effect of teacher perceptions on student performance when SCMATH 
$=4$, and $a_{1} b_{1}$ is the conditional indirect effect of interest. Equation 3, much simpler to compute by hand than the $S E$ for Model 3 (used in Equation 20), yields the $S E$ of this conditional indirect effect. A percentile-based bootstrap CI can be obtained readily using one of several existing statistical programming languages. Alternatively, the SEs and bootstrap CIs can be obtained using existing SEM software with bootstrapping capabilities, such as AMOS or Mplus. With the aid of the diagrams in Figure 2, conditional indirect effects can be estimated using the moderator centering approach for any of the models we just discussed. However, we caution that the indirect effects reported by SEM software use only the first-order variance; if the second-order variance is desired, hand calculations will be necessary. In addition, this approach involves computing a new variable and estimating parameters separately for every conditional value (or combination of values) of interest. Moreover, this approach is useful only to the extent that meaningful, nonarbitrary values of the moderator(s) can be identified a priori. Finally, this method cannot be used for identifying regions of significance via the $\mathrm{J}-\mathrm{N}$ technique.

\section{Graphical Illustration of Conditional Indirect Effects}

We have already described how regions of significance and confidence bands may be employed to illustrate conditional indirect effects. Tate (1998) described some creative ways to graphically illustrate indirect effects in complex path models. Simple path diagram representations are given by decomposing interactive effects into simple slopes. Thus, Model 4 (in Figure 2, Panel D) could be represented more simply, as in Figure 4, by including only simple slopes and no product terms. The intuitive appeal of such diagrams makes them more appropriate for communicating results than the diagrams we provide in Figure 2, which are more suitable for aiding in model specification. A limitation of diagrams such as the one in Figure 4 is that they cannot easily accommodate all parameter estimates; the variances and covariances associated with interaction terms are not included, but these are rarely of substantive interest anyway.

\section{DISCUSSION}

In this article we have attempted to dispel confusion about moderated mediation, a collection of effects with sometimes conflicting or overlapping definitions, by considering all such effects as special cases of moderated regression (or, more generally, path analysis or SEM). We unify such effects under the rubric conditional indirect effects. This terminology reflects the fact that the effect of interest is an indirect (mediation) effect that is potentially conditional on the value of one or more moderators. We also detail two general approaches 


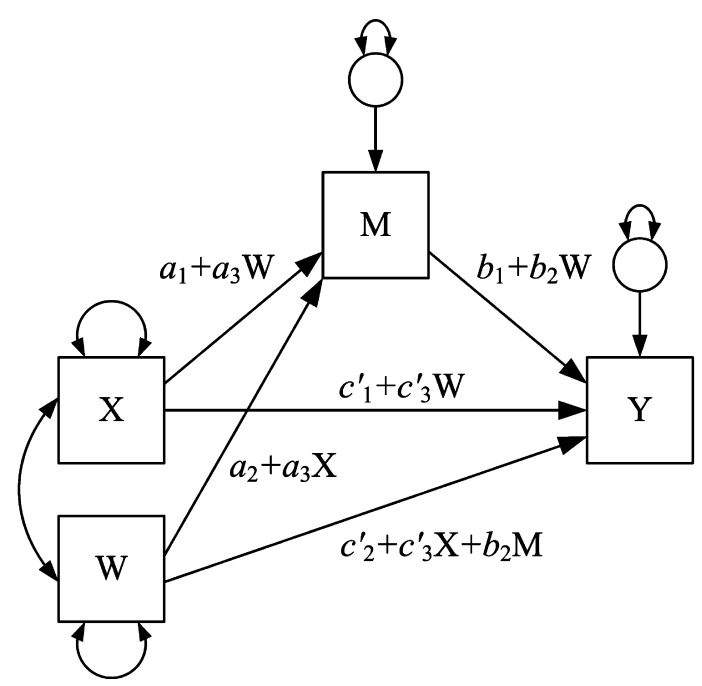

FIGURE 4 A compact way to express parameter estimates in models containing conditional indirect effects, after Tate (1998).

to estimating and determining the significance of conditional indirect effects, one using resampling to construct asymmetric CIs and one using the first- and second-order multivariate delta method to derive SEs and construct CIs. The latter method can also be used to determine values of the moderator(s) for which indirect effects are significant (the J-N technique) if the total sample size is large. Finally, we provide an SPSS macro to assist in implementing these procedures in practice. We advocate bootstrapping indirect effects whenever circumstances permit it, but it may be wise to use both approaches in empirical applications, given the advantages associated with each.

In using these methods, researchers should be aware of model assumptions. Tests of indirect effects are applications of multiple regression. Researchers should be mindful that assumptions are not egregiously violated in practice. The most important assumptions of these analyses are that the model is correctly specified to a reasonable degree (i.e., relationships are linear, disturbances are normally distributed, homoscedastic, and independent, and no important variables have been omitted; see Cohen, Cohen, West, \& Aiken, 2003). If bootstrapping is used, the only assumptions required when testing conditional indirect effects are linearity of the relationships in the system and independence of the observations. In addition, if SEM is used and any data are missing, they should be missing at random (MAR) or missing completely at random (MCAR) in order to satisfy maximum likelihood assumptions (Arbuckle, 1996). Furthermore, 
these models are causal models, so any conclusions rest on the assumptions that causally linked variables are characterized by temporal precedence, concomitant variation, and elimination of sources of spurious correlation. Cole and Maxwell (2003) and MacKinnon et al. (2002) discussed many issues relevant to study design and model specification that should be considered by anyone planning to assess indirect effects, conditional or otherwise.

Finally, it is important to keep in mind the recommendations of many methodologists (e.g., Wilkinson \& the APA Task Force on Statistical Inference, 1999) concerning the need for increased emphasis on confidence intervals. CIs imply the result of any point hypothesis test and give additional information on the precision of estimates of conditional indirect effects. Both methods described here permit the construction of CIs-symmetric intervals in the case of the normaltheory approach (using first- or second-order $S E$ s) and asymmetric intervals in the case of resampling. In general, asymmetric intervals are preferable because they explicitly incorporate information on the skew of product distributions.

As researchers' hypotheses grow more specific, such as those involving conditional indirect effects, the ability to evaluate hypotheses will be limited by the sophistication of available statistical tests. In recognition of this, our ultimate intention has been to provide an intuitive guide to properly construing such tests and providing an accessible means for researchers to conduct analyses of conditional indirect effects. It should be remembered that the normal-theory and bootstrap methods likely differ in power. Some research has been undertaken with respect to the power of tests of simple mediation (MacKinnon et al., 2004), and our own simulation addressed power under a set of limited conditions. Investigating the power to detect conditional indirect effects would be an interesting direction for future research.

\section{ACKNOWLEDGMENTS}

This research used the Study of Life Transitions: 1983-1985 data set (made accessible in 1996, machine-readable data files). These data were collected by Jacquelynne Eccles and made available through the archive of the Henry A. Murray Research Center of The Radcliffe Institute for Advanced Study, Harvard University, Cambridge, Massachusetts.

This work was funded in part by National Institute on Drug Abuse Grant DA16883 awarded to the first author while at the University of North Carolina at Chapel Hill. We thank $\mathrm{Li}$ Cai for valuable input regarding derivations in the Technical Appendix, Stephanie Madon and Courtney Stevens for help with the applied example, and Daniel J. Bauer for helpful advice in improving the manuscript. The SPSS macro syntax is available online through http://www.quantpsy.org/. 


\section{REFERENCES}

Aiken, L. S., \& West, S. G. (1991). Multiple regression: Testing and interpreting interactions. Newbury Park, CA: Sage.

Arbuckle, J. L. (1996). Full information estimation in the presence of incomplete data. In G. A. Marcoulides \& R. E. Schumacker (Eds.), Advanced structural equation modeling: Issues and techniques. Mahwah, NJ: Lawrence Erlbaum Associates, Inc.

Aroian, L. A. (1947). The probability function of the product of two normally distributed variables. Annals of Mathematical Statistics, 18, 265-271.

Baron, R. M., \& Kenny, D. A. (1986). The moderator-mediator variable distinction in social psychological research: Conceptual, strategic, and statistical considerations. Journal of Personality \& Social Psychology, 51, 1173-1182.

Bauer, D. J., \& Curran, P. J. (2005). Probing interactions in fixed and multilevel regression: Inferential and graphical techniques. Multivariate Behavioral Research, 40, 373-400.

Bauer, D. J., Preacher, K. J., \& Gil, K. M. (2006). Conceptualizing and testing random indirect effects and moderated mediation in multilevel models: New procedures and recommendations. Psychological Methods, 11, 142-163.

Bollen, K. A. (1987). Total, direct, and indirect effects in structural equation models. In C. C. Clogg (Ed.), Sociological methodology 1987 (pp. 37-69). Washington, DC: American Sociological Association.

Bollen, K. A. (1989). Structural equations with latent variables. New York: Wiley.

Bollen, K. A., \& Stine, R. (1990). Direct and indirect effects: Classical and bootstrap estimates of variability. Sociological Methodology, 20, 115-140.

Calvete, E., \& Cardenoso, O. (2005). Gender differences in cognitive vulnerability to depression and behavior problems in adolescents. Journal of Abnormal Child Psychology, 33, 179-192.

Cohen, J., Cohen, P., West, S. G., \& Aiken, L. S. (2003). Applied multiple regression/correlation analysis for the behavioral sciences (3rd ed.). Mahwah, NJ: Lawrence Erlbaum Associates, Inc.

Cole, D. A., \& Maxwell, S. E. (2003). Testing mediational models with longitudinal data: Questions and tips in the use of structural equation modeling. Journal of Abnormal Psychology, 112, 558577.

Craig, C. C. (1936). On the frequency function of xy. Annals of Mathematical Statistics, 7, 1-15.

Darlington, R. B. (1990). Regression and linear models. New York: McGraw-Hill.

Donaldson, S. I. (2001). Mediator and moderator analysis in program development. In S. Sussman (Ed.), Handbook of program development for health behavior research and practice (pp. 470496). Thousand Oaks, CA: Sage.

Eccles, J. S. (1988). Achievement beliefs and the environment (Rep. No. NICHD-PS-019736). Bethesda, MD: National Institute of Child Health and Development (National Institute of Health).

Efron, B. (1987). Better bootstrap confidence intervals. Journal of the American Statistical Association, 82, 171-185.

Efron, B., \& Tibshirani, R. J. (1998). An introduction to the bootstrap. Boca Raton, FL: Chapman $\&$ Hall.

Fredrickson, B. L., Tugade, M. M., Waugh, C. E., \& Larkin, G. R. (2003). What good are positive emotions in crises? A prospective study of resilience and emotions following the terrorist attacks on the United States on September 11th, 2001. Journal of Personality and Social Psychology, $84,365-376$.

Frone, M. R. (1999). Work stress and alcohol use. Alcohol Research \& Health, 23, 284-291.

Goodman, L. A. (1960). On the exact variance of products. Journal of the American Statistical Association, 55, 708-713.

Graybill, F. A. (1983). Matrices with applications in statistics (2nd ed.). Belmont, CA: Duxbury. 
Hodges, E. V. E., \& Perry, D. G. (1999). Personal and interpersonal antecedents and consequences of victimization by peers. Journal of Personality and Social Psychology, 76, 677-685.

Holmbeck, G. N. (1997). Toward terminological, conceptual, and statistical clarity in the study of mediators and moderators: Examples from the child-clinical and pediatric psychology literatures. Journal of Consulting and Clinical Psychology, 65, 599-610.

James, L. R., \& Brett, J. M. (1984). Mediators, moderators, and tests for mediation. Journal of Applied Psychology, 69, 307-321.

Johnson, P. O., \& Neyman, J. (1936). Tests of certain linear hypotheses and their applications to some educational problems. Statistical Research Memoirs, 1, 57-93.

Judd, C. M., \& Kenny, D. A. (1981). Process analysis: Estimating mediation in treatment evaluations. Evaluation Review, 5, 602-619.

Judd, C. M., Kenny, D. A., \& McClelland, G. H. (2001). Estimating and testing mediation and moderation in within-subject designs. Psychological Methods, 6, 115-134.

Judd, C. M., \& McClelland, G. H. (1989). Data analysis: A model comparison approach. San Diego: Harcourt, Brace, Jovanovich.

Lambert, A. J., Payne, B. K., Jacoby, L. L., Shaffer, L. M., Chasteen, A. L., \& Khan, S. R. (2003). Stereotypes as dominant responses: On the "social facilitation" of prejudice in anticipated public contexts. Journal of Personality and Social Psychology, 84, 277-295.

Lockwood, C. M., \& MacKinnon, D. P. (1998). Bootstrapping the standard error of the mediated effect. Proceedings of the 23rd annual meeting of SAS Users Group International (pp. 997-1002). Cary, NC: SAS Institute, Inc.

Lunneborg, C. A. (2000). Data analysis by resampling: Concepts and applications. Pacific Grove, CA: Duxbury.

MacKinnon, D. P. (1992). Statistical simulations using the CALIS procedure. Proceedings of the 17th Annual SAS Users Group International Conference 1992 (pp. 1199-1203). Cary, NC: SAS Institute, Inc.

MacKinnon, D. P. (2000). Contrasts in multiple mediator models. In J. S. Rose, L. Chassin, C. C. Presson, \& S. J. Sherman (Eds.), Multivariate applications in substance use research (pp. 141160). Mahwah, NJ: Lawrence Erlbaum Associates, Inc.

MacKinnon, D. P. (2001). Commentary on Donaldson, Mediator and moderator analysis in program development. In S. Sussman (Ed.), Handbook of program development for health behavior research and practice (pp. 497-500). Thousand Oaks, CA: Sage.

MacKinnon, D. P., Lockwood, C. M., \& Hoffman, J. M. (1998, June). A new method to test for mediation. Paper presented at the annual meeting of the Society for Prevention Research, Park City, UT.

MacKinnon, D. P., Lockwood, C. M., Hoffman, J. M., West, S. G., \& Sheets, V. (2002). A comparison of methods to test mediation and other intervening variable effects. Psychological Methods, 7, $83-104$

MacKinnon, D. P., Lockwood, C. M., \& Williams, J. (2004). Confidence limits for the indirect effect: Distribution of the product and resampling methods. Multivariate Behavioral Research, $39,99-128$

MacKinnon, D. P., Warsi, G., \& Dwyer, J. H. (1995). A simulation study of mediated effect measures. Multivariate Behavioral Research, 30, 41-62.

Madon, S., Guyll, M., Spoth, R. L., Cross, S. E., \& Hilbert, S. J. (2003). The self-fulfilling influence of mother expectations on children's underage drinking. Journal of Personality and Social Psychology, 84, 1188-1205.

Madon, S., Jussim, L., \& Eccles, J. (1997). In search of the powerful self-fulfilling prophecy. Journal of Personality and Social Psychology, 72, 791-809.

Mandel, N., \& Johnson, E. J. (2002). When web pages influence choice: Effects of visual primes on experts and novices. Journal of Consumer Research, 29, 235-245. 
Mood, A., Graybill, F. A., \& Boes, D. C. (1974). Introduction to the theory of statistics. New York: McGraw-Hill.

Morgan-Lopez, A. A. (2003). A simulation study of the mediated baseline by treatment interaction effect in preventive intervention trials. Unpublished doctoral dissertation, Arizona State University.

Morgan-Lopez, A. A., \& MacKinnon, D. P. (2006). Demonstration and evaluation of a method for assessing mediated moderation. Behavior Research Methods, 38, 77-87.

Morgan-Lopez, A. A., Castro, F. G., Chassin, L., \& MacKinnon, D. P. (2003). A mediated moderation model of cigarette use among Mexican American youth. Addictive Behaviors, 28, 583-589.

Muller, D., Judd, C. M., \& Yzerbyt, V. Y. (2005). When moderation is mediated and mediation is moderated. Journal of Personality and Social Psychology, 89, 852-863.

Murray, S. L., Bellavia, G. M., Rose, P., \& Griffin, D. W. (2003). Once hurt, twice hurtful: How perceived regard regulates daily marital interactions. Journal of Personality and Social Psychology, $84,126-147$.

Pothoff, R. F. (1964). On the Johnson-Neyman technique and some extensions thereof. Psychometrika, 29, 241-256.

Preacher, K. J., Curran, P. J., \& Bauer, D. J. (2003, September). Probing interactions in multiple linear regression, latent curve analysis, and hierarchical linear modeling: Interactive calculation tools for establishing simple intercepts, simple slopes, and regions of significance [Computer software]. Available from http://www.quantpsy.org/

Preacher, K. J., Curran, P. J., \& Bauer, D. J. (2006). Computational tools for probing interaction effects in multiple linear regression, multilevel modeling, and latent curve analysis. Journal of Educational and Behavioral Statistics, 31, 437-448.

Preacher, K. J., \& Hayes, A. F. (2004). SPSS and SAS procedures for estimating indirect effects in simple mediation models. Behavior Research Methods, Instruments, \& Computers, 36, 717 731.

Preacher, K. J., \& Hayes, A. F. (2006). Asymptotic and resampling strategies for assessing and comparing indirect effects in simple and multiple mediator models. Manuscript submitted for publication.

Rogosa, D. (1981). On the relationship between the Johnson-Neyman region of significance and statistical tests of parallel within group regressions. Educational and Psychological Measurement, $41,73-84$.

Rose, B. M., Holmbeck, G. N., Coakley, R. M., \& Franks, E. A. (2004). Mediator and moderator effects in developmental and behavioral pediatric research. Developmental and Behavioral Pediatrics, 25, 58-67.

Shrout, P. E., \& Bolger, N. (2002). Mediation in experimental and nonexperimental studies: New procedures and recommendations. Psychological Methods, 7, 422-445.

Slater, M. D., Hayes, A. F., \& Ford, V. (in press). Examining the moderating and mediating effects of media and individual differences on adolescents' perceptions of alcohol-related risks. Communication Research.

Sobel, M. E. (1982). Asymptotic confidence intervals for indirect effects in structural equations models. In S. Leinhart (Ed.), Sociological methodology 1982 (pp. 290-312). San Francisco: Jossey-Bass.

Sobel, M. E. (1986). Some new results on indirect effects and their standard errors in covariance structure models. In N. Tuma (Ed.), Sociological methodology 1986 (pp. 159-186). Washington, DC: American Sociological Association.

Stine, R. (1989). An introduction to bootstrap methods. Sociological Methods and Research, 18, 243-291.

Stolzenberg, R. M. (1980). The measurement and decomposition of causal effects in nonlinear and nonadditive models. Sociological Methodology, 11, 459-488. 
Stone, C. A., \& Sobel, M. E. (1990). The robustness of estimates of total indirect effects in covariance structure models estimated by maximum likelihood. Psychometrika, 55, 337-352.

Tate, R. L. (1998). Effect decomposition in interaction and nonlinear models. In R. E. Schumacker \& G. A. Marcoulides (Eds.), Interaction and nonlinear effects in structural equation modeling. Mahwah, NJ: Lawrence Erlbaum Associates, Inc.

Tein, J.-Y., Sandler, I. N., MacKinnon, D. P., \& Wolchik, S. A. (2004). How did it work? Who did it work for? Mediation in the context of a moderated prevention effect for children of divorce. Journal of Consulting and Clinical Psychology, 72, 617-624.

Tein, J.-Y., Sandler, I. N., \& Zautra, A. J. (2000). Stressful life events, psychological distress, coping, and parenting of divorced mothers: A longitudinal study. Journal of Family Psychology, 14, 27-41.

Wegener, D. T., \& Fabrigar, L. R. (2000). Analysis and design for nonexperimental data: Addressing causal and noncausal hypotheses. In H. T. Reis \& C. M. Judd (Eds.), Handbook of research methods in social and personality psychology (pp. 412-450). New York: Cambridge University Press.

Wilkinson, L., \& the APA Task Force on Statistical Inference. (1999). Statistical methods in psychology journals: Guidelines and explanations. American Psychologist, 54, 594-604.

\section{TECHNICAL APPENDIX}

This appendix contains derivations of point estimates and standard errors of conditional indirect effects for all five models presented in Figure 2. Both firstorder and second-order estimates are provided.

\section{Part I: Point Estimates of Conditional Indirect Effects}

We use Model 1 (when $X$ acts as a moderator of the $b$ path) as an example, although the same method may be applied to any of the models depicted in Figure 2. The indirect effect of $X$ on $Y$ in Model 1 can be easily derived using a matrix algebra approach described by Sobel (1986) and Bollen $(1987,1989)$. The path coefficients (direct effects) linking pairs of variables in Figure 2, Panel A are represented in the matrix $\mathbf{B}$ (here and throughout, matrices and vectors are represented in boldface type). The rows and columns of $\mathbf{B}$ correspond to all of the variables in the system, such that each element represents the effect of the column variable on the row variable. In the present case,

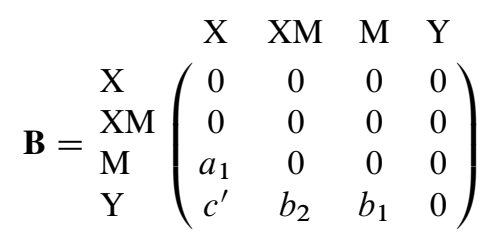

and $a_{1}, c^{\prime}$, and so on are regression weights or path coefficients. This $\mathbf{B}$ matrix may be collapsed by noting that XM is an exact function of variables already 
represented. Effects of individual variables can be obtained by computing partial derivatives of the reduced-form equations of endogenous (dependent) variables, yielding a more compact effect matrix $\mathbf{B}^{*}$ :

$$
\mathbf{B}^{*}=\underset{\mathrm{Y}}{\mathrm{X}}\left(\begin{array}{ccc}
\mathrm{X} & \mathrm{M} & \mathrm{Y} \\
0 & 0 & 0 \\
a_{1} & 0 & 0 \\
c^{\prime}+b_{2} M & b_{1}+b_{2} X & 0
\end{array}\right)
$$

The indirect effects of all variables on all other variables can be obtained using the formula:

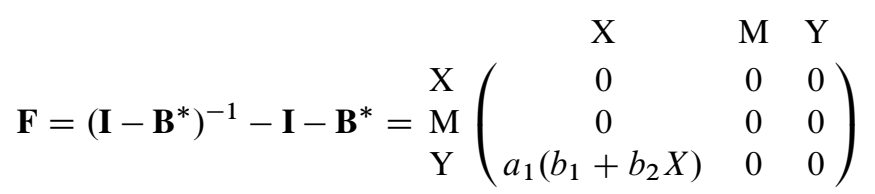

The point estimate of the indirect effect of $X$ on $Y$ is thus $f(\hat{\boldsymbol{\theta}} \mid X)=\hat{a}_{1}\left(\hat{b}_{1}+\right.$ $\left.\hat{b}_{2} X\right),{ }^{8}$ where $\hat{\boldsymbol{\theta}}$ is a vector containing the relevant random variables, here the sample regression coefficients $\hat{a}_{1}, \hat{b}_{1}$, and $\hat{b}_{2}$.

\section{Part II: First- and Second-Order Variance Approximations}

This section contains derivations of first- and second-order variance approximations for the simple mediation model (Model 0) and Models 1-5 using the second-order delta method (cf. Mood et al., 1974). Bollen (1987, 1989) and Sobel (1982) demonstrate the use of the first-order multivariate delta method in determining $S E$ s of indirect effects. Letting $\mathbf{D}=\left.\partial_{\hat{\boldsymbol{\theta}}} f(\hat{\boldsymbol{\theta}})\right|_{\boldsymbol{\mu}}$ (i.e., a vector of derivatives of $f$ with respect to each random variable), the $S E$ for the indirect effect is obtained by computing the square root of the variance of $f(\hat{\boldsymbol{\theta}})$, given by:

$$
\operatorname{var}[f(\hat{\boldsymbol{\theta}})]=\left.\left\{\mathbf{D}^{\prime} \hat{\boldsymbol{\Sigma}}(\hat{\boldsymbol{\theta}}) \mathbf{D}\right\}\right|_{\boldsymbol{\mu}}
$$

where, $\boldsymbol{\mu}=E(\hat{\boldsymbol{\theta}})$ and $\hat{\boldsymbol{\Sigma}}(\hat{\boldsymbol{\theta}})$ is the sample estimate of the asymptotic covariance matrix of $\hat{\boldsymbol{\theta}}$. The $\hat{\boldsymbol{\Sigma}}(\hat{\boldsymbol{\theta}})$ matrix is typically available by request in most regression and SEM software applications. In the following derivations,

$\hat{\boldsymbol{\theta}}$ is a column vector of the sample regression coefficients involved in an indirect effect.

\footnotetext{
${ }^{8}$ This indirect effect, and the others we discussed, may also be obtained via the chain rule from elementary calculus (Stolzenberg, 1980). For example: $\frac{\partial Y}{\partial X}=\frac{\partial Y}{\partial M} \cdot \frac{\partial M}{\partial X}=\left(b_{1}+b_{2} X\right) \cdot a_{1}$.
} 
$\boldsymbol{\mu}$ is a column vector of the means of the regression coefficients, i.e., $\boldsymbol{\mu}=$ $E[\hat{\boldsymbol{\theta}}]$.

$f(\hat{\boldsymbol{\theta}})$ is the effect of interest, a differentiable function of the coefficients in $\hat{\theta}$.

$\hat{\boldsymbol{\Sigma}}(\hat{\boldsymbol{\theta}})$ is the covariance matrix of $\hat{\boldsymbol{\theta}}$.

$\mathbf{D}=\partial_{\boldsymbol{\theta}} f(\hat{\boldsymbol{\theta}})$ is the gradient of $f(\hat{\boldsymbol{\theta}})$ evaluated at $\boldsymbol{\mu}$.

$\mathbf{H}=\partial_{\hat{\boldsymbol{\theta}}}^{2} f(\hat{\boldsymbol{\theta}})$ is the Hessian of $f(\hat{\boldsymbol{\theta}})$ evaluated at $\boldsymbol{\mu}$.

The gradient and Hessian are, respectively, matrices of first and second partial derivatives of $f(\hat{\boldsymbol{\theta}})$ with respect to all relevant free parameters. For example, in simple mediation we have:

$$
\begin{aligned}
\hat{\boldsymbol{\theta}} & =\left[\begin{array}{ll}
\hat{a}_{1} & \hat{b}_{1}
\end{array}\right]^{\prime} \\
\boldsymbol{\mu} & =\left[\begin{array}{ll}
a_{1} & b_{1}
\end{array}\right]^{\prime} \\
f(\hat{\boldsymbol{\theta}}) & =\hat{a}_{1} \hat{b}_{1} \\
\mathbf{D} & =\left.\partial_{\boldsymbol{\theta}} f(\hat{\boldsymbol{\theta}})\right|_{\boldsymbol{\mu}} \\
\mathbf{H} & =\left.\left(\begin{array}{ll}
\partial_{a_{1} a_{1}}^{2} f(\hat{\boldsymbol{\theta}}) & \partial_{a_{1} b_{1}}^{2} f(\hat{\boldsymbol{\theta}}) \\
\partial_{a_{1} b_{1}}^{2} f(\hat{\boldsymbol{\theta}}) & \partial_{b_{1} b_{1}}^{2} f(\hat{\boldsymbol{\theta}})
\end{array}\right)\right|_{\boldsymbol{\mu}}
\end{aligned}
$$

Because $\operatorname{var}[f(\hat{\boldsymbol{\theta}})]=E\left[f^{2}(\hat{\boldsymbol{\theta}})\right]-E^{2}[f(\hat{\boldsymbol{\theta}})]$, we need $f^{2}(\hat{\boldsymbol{\theta}})$ and $E[f(\hat{\boldsymbol{\theta}})]$.

$$
f(\hat{\boldsymbol{\theta}}) \approx f(\boldsymbol{\mu})+\mathbf{D}^{\prime}(\hat{\boldsymbol{\theta}}-\boldsymbol{\mu})+\frac{1}{2}(\hat{\boldsymbol{\theta}}-\boldsymbol{\mu})^{\prime} \mathbf{H}(\hat{\boldsymbol{\theta}}-\boldsymbol{\mu})
$$

(by Taylor's theorem)

$$
\begin{aligned}
f^{2}(\hat{\boldsymbol{\theta}}) \approx & \left(f(\boldsymbol{\mu})+\mathbf{D}^{\prime}(\hat{\boldsymbol{\theta}}-\boldsymbol{\mu})+\frac{1}{2}(\hat{\boldsymbol{\theta}}-\boldsymbol{\mu})^{\prime} \mathbf{H}(\hat{\boldsymbol{\theta}}-\boldsymbol{\mu})\right)^{2} \\
= & f^{2}(\boldsymbol{\mu})+\mathbf{D}^{\prime}(\hat{\boldsymbol{\theta}}-\boldsymbol{\mu})(\hat{\boldsymbol{\theta}}-\boldsymbol{\mu})^{\prime} \mathbf{D} \\
& +\frac{1}{4}(\hat{\boldsymbol{\theta}}-\boldsymbol{\mu})^{\prime} \mathbf{H}(\hat{\boldsymbol{\theta}}-\boldsymbol{\mu})(\hat{\boldsymbol{\theta}}-\boldsymbol{\mu})^{\prime} \mathbf{H}(\hat{\boldsymbol{\theta}}-\boldsymbol{\mu}) \\
& +2 f(\boldsymbol{\mu}) \mathbf{D}^{\prime}(\hat{\boldsymbol{\theta}}-\boldsymbol{\mu})+f(\boldsymbol{\mu})(\hat{\boldsymbol{\theta}}-\boldsymbol{\mu})^{\prime} \mathbf{H}(\hat{\boldsymbol{\theta}}-\boldsymbol{\mu}) \\
& +\mathbf{D}^{\prime}(\hat{\boldsymbol{\theta}}-\boldsymbol{\mu})(\hat{\boldsymbol{\theta}}-\boldsymbol{\mu})^{\prime} \mathbf{H}(\hat{\boldsymbol{\theta}}-\boldsymbol{\mu})
\end{aligned}
$$


PREACHER, RUCKER, HAYES

$$
\begin{aligned}
& E\left[f^{2}(\hat{\boldsymbol{\theta}})\right] \approx E\left[\begin{array}{c}
f^{2}(\boldsymbol{\mu})+\mathbf{D}^{\prime}(\hat{\boldsymbol{\theta}}-\boldsymbol{\mu})(\hat{\boldsymbol{\theta}}-\boldsymbol{\mu})^{\prime} \mathbf{D} \\
+ \\
+\frac{1}{4}(\hat{\boldsymbol{\theta}}-\boldsymbol{\mu})^{\prime} \mathbf{H}(\hat{\boldsymbol{\theta}}-\boldsymbol{\mu})(\hat{\boldsymbol{\theta}}-\boldsymbol{\mu})^{\prime} \mathbf{H}(\hat{\boldsymbol{\theta}}-\boldsymbol{\mu}) \\
+2 f(\boldsymbol{\mu}) \mathbf{D}^{\prime}(\hat{\boldsymbol{\theta}}-\boldsymbol{\mu})+f(\boldsymbol{\mu})(\hat{\boldsymbol{\theta}}-\boldsymbol{\mu})^{\prime} \mathbf{H}(\hat{\boldsymbol{\theta}}-\boldsymbol{\mu}) \\
+\mathbf{D}^{\prime}(\hat{\boldsymbol{\theta}}-\boldsymbol{\mu})(\hat{\boldsymbol{\theta}}-\boldsymbol{\mu})^{\prime} \mathbf{H}(\hat{\boldsymbol{\theta}}-\boldsymbol{\mu})
\end{array}\right] \\
&= f^{2}(\boldsymbol{\mu})+\mathbf{D}^{\prime} \hat{\boldsymbol{\Sigma}}(\hat{\boldsymbol{\theta}}) \mathbf{D}+\frac{1}{4} E\left[(\hat{\boldsymbol{\theta}}-\boldsymbol{\mu})^{\prime} \mathbf{H}(\hat{\boldsymbol{\theta}}-\boldsymbol{\mu})(\hat{\boldsymbol{\theta}}-\boldsymbol{\mu})^{\prime} \mathbf{H}(\hat{\boldsymbol{\theta}}-\boldsymbol{\mu})\right] \\
&+f(\boldsymbol{\mu}) \operatorname{tr}\{\mathbf{H} \hat{\boldsymbol{\Sigma}}(\hat{\boldsymbol{\theta}})\} \\
&= f^{2}(\boldsymbol{\mu})+\mathbf{D}^{\prime} \hat{\boldsymbol{\Sigma}}(\hat{\boldsymbol{\theta}}) \mathbf{D}+\frac{1}{4}(\operatorname{tr}\{\mathbf{H} \hat{\boldsymbol{\Sigma}}(\hat{\boldsymbol{\theta}})\})^{2}+\frac{1}{2} \operatorname{tr}\left\{(\mathbf{H} \hat{\boldsymbol{\Sigma}}(\hat{\boldsymbol{\theta}}))^{2}\right\} \\
&+f(\boldsymbol{\mu}) \operatorname{tr}\{\mathbf{H} \hat{\boldsymbol{\Sigma}}(\hat{\boldsymbol{\theta}})\}
\end{aligned}
$$

(by Graybill's (1983) Theorem 10.9.10)

$$
\begin{aligned}
E[f(\hat{\boldsymbol{\theta}})] \approx & E\left[f(\boldsymbol{\mu})+\mathbf{D}^{\prime}(\hat{\boldsymbol{\theta}}-\boldsymbol{\mu})+\frac{1}{2}(\hat{\boldsymbol{\theta}}-\boldsymbol{\mu})^{\prime} \mathbf{H}(\hat{\boldsymbol{\theta}}-\boldsymbol{\mu})\right] \\
= & f(\boldsymbol{\mu})+\mathbf{D}^{\prime} \underbrace{E[(\hat{\boldsymbol{\theta}}-\boldsymbol{\mu})]}_{0}+\frac{1}{2} E\left[(\hat{\boldsymbol{\theta}}-\boldsymbol{\mu})^{\prime} \mathbf{H}(\hat{\boldsymbol{\theta}}-\boldsymbol{\mu})\right] \\
= & f(\boldsymbol{\mu})+\frac{1}{2} \operatorname{tr}\{\mathbf{H} \hat{\boldsymbol{\Sigma}}(\hat{\boldsymbol{\theta}})\} \\
\operatorname{var}[f(\hat{\boldsymbol{\theta}}) \approx & E\left[f^{2}(\hat{\boldsymbol{\theta}})\right]-E^{2}[f(\hat{\boldsymbol{\theta}})] \\
= & f^{2}(\boldsymbol{\mu})+\mathbf{D}^{\prime} \hat{\boldsymbol{\Sigma}}(\hat{\boldsymbol{\theta}}) \mathbf{D}+\frac{1}{4}(\operatorname{tr}\{\mathbf{H} \hat{\boldsymbol{\Sigma}}(\hat{\boldsymbol{\theta}})\})^{2}+\frac{1}{2} \operatorname{tr}\left\{(\mathbf{H} \hat{\boldsymbol{\Sigma}}(\hat{\boldsymbol{\theta}}))^{2}\right\} \\
& +f(\boldsymbol{\mu}) \operatorname{tr}\{\mathbf{H} \hat{\boldsymbol{\Sigma}}(\hat{\boldsymbol{\theta}})\}-\left(f(\boldsymbol{\mu})+\frac{1}{2} \operatorname{tr}\{\mathbf{H} \hat{\boldsymbol{\Sigma}}(\hat{\boldsymbol{\theta}})\}\right)^{2} \\
= & f^{2}(\boldsymbol{\mu})+\mathbf{D}^{\prime} \hat{\boldsymbol{\Sigma}}(\hat{\boldsymbol{\theta}}) \mathbf{D}+\frac{1}{4}(\operatorname{tr}\{\mathbf{H} \hat{\boldsymbol{\Sigma}}(\hat{\boldsymbol{\theta}})\})^{2}+\frac{1}{2} \operatorname{tr}\left\{(\mathbf{H} \hat{\boldsymbol{\Sigma}}(\hat{\boldsymbol{\theta}}))^{2}\right\} \\
& +f(\boldsymbol{\mu}) \operatorname{tr}\{\mathbf{H} \hat{\boldsymbol{\Sigma}}(\hat{\boldsymbol{\theta}})\}-f^{2}(\boldsymbol{\mu})-f(\boldsymbol{\mu}) \operatorname{tr}\{\mathbf{H} \hat{\boldsymbol{\Sigma}}(\hat{\boldsymbol{\theta}})\}-\frac{1}{4}(\operatorname{tr}\{\mathbf{H} \hat{\boldsymbol{\Sigma}}(\hat{\boldsymbol{\theta}})\})^{2} \\
= & \underbrace{\mathbf{D}^{\prime} \hat{\boldsymbol{\Sigma}}(\hat{\boldsymbol{\theta}}) \mathbf{D}}_{\text {first-order part }}+\underbrace{\frac{1}{2} \operatorname{tr}\left\{(\mathbf{H} \hat{\boldsymbol{\Sigma}}(\hat{\boldsymbol{\theta}}))^{2}\right\}}_{\text {second-order part }}
\end{aligned}
$$


The second-order approximation $\operatorname{var}[f(\hat{\boldsymbol{\theta}})] \approx \mathbf{D}^{\prime} \hat{\boldsymbol{\Sigma}}(\hat{\boldsymbol{\theta}}) \mathbf{D}+\frac{1}{2} \operatorname{tr}\left\{(\mathbf{H} \hat{\boldsymbol{\Sigma}}(\hat{\boldsymbol{\theta}}))^{2}\right\}$ yields the exact variance of the conditional indirect effect for all models considered here, assuming normality.

Model 0: Simple Mediation

$$
\begin{aligned}
& \hat{\boldsymbol{\theta}}=\left[\begin{array}{ll}
\hat{a}_{1} & \hat{b}_{1}
\end{array}\right]^{\prime} \quad f(\hat{\boldsymbol{\theta}})=\hat{a}_{1} \hat{b}_{1} \quad \hat{\boldsymbol{\Sigma}}(\hat{\boldsymbol{\theta}})=\left[\begin{array}{cc}
s_{\hat{a}_{1}}^{2} & 0 \\
0 & s_{\hat{b}_{1}}^{2}
\end{array}\right] \quad \mathbf{H}=\left[\begin{array}{ll}
0 & 1 \\
1 & 0
\end{array}\right] \\
& \operatorname{var}[f(\hat{\boldsymbol{\theta}})] \approx \mathbf{D}^{\prime} \hat{\boldsymbol{\Sigma}}(\hat{\boldsymbol{\theta}}) \mathbf{D}+\frac{1}{2} \operatorname{tr}\left\{(\mathbf{H} \hat{\boldsymbol{\Sigma}}(\hat{\boldsymbol{\theta}}))^{2}\right\} \\
&=\underbrace{\hat{b}_{1}^{2} s_{\hat{a}_{1}}^{2}+\hat{a}_{1}^{2} s_{\hat{b}_{1}}^{2}}_{\text {first-order approximation }}+\underbrace{s_{\hat{a}_{1}}^{2} s_{\hat{b}_{1}}^{2}}_{\begin{array}{c}
\text { additional part from second-order } \\
\text { approximation (exact variance) }
\end{array}}
\end{aligned}
$$

(Note: This result is also due to MacKinnon et al. (1995) using the secondorder delta method described by Mood et al. (1974, p. 534). Aroian (1947) and Goodman (1960) provide alternative derivations converging on the same result.)

Model 1: When the Independent Variable Is Also the Moderator

$$
\begin{aligned}
\hat{\boldsymbol{\theta}}= & {\left[\begin{array}{lll}
\hat{a}_{1} & \hat{b}_{1} & \hat{b}_{2}
\end{array}\right]^{\prime} \quad f(\hat{\boldsymbol{\theta}} \mid X)=\hat{a}_{1}\left(\hat{b}_{1}+\hat{b}_{2} X\right) } \\
\hat{\boldsymbol{\Sigma}}(\hat{\boldsymbol{\theta}})= & {\left[\begin{array}{ccc}
s_{\hat{a}_{1}}^{2} & 0 & 0 \\
0 & s_{\hat{b}_{1}}^{2} & s_{\hat{b}_{1}, \hat{b}_{2}} \\
0 & s_{\hat{b}_{1}, \hat{b}_{2}} & s_{\hat{b}_{2}}^{2}
\end{array}\right] \quad \mathbf{H}=\left[\begin{array}{ccc}
0 & 1 & X \\
1 & 0 & 0 \\
X & 0 & 0
\end{array}\right] } \\
\operatorname{var}[f(\hat{\boldsymbol{\theta}} \mid X)] \approx & \mathbf{D}^{\prime} \hat{\boldsymbol{\Sigma}}(\hat{\boldsymbol{\theta}}) \mathbf{D}+\frac{1}{2} \operatorname{tr}\left\{(\mathbf{H} \hat{\boldsymbol{\Sigma}}(\hat{\boldsymbol{\theta}}))^{2}\right\} \\
= & \underbrace{\left(\hat{b}_{1}+\hat{b}_{2} X\right)^{2} s_{\hat{a}_{1}}^{2}+\hat{a}_{1}^{2}\left(s_{\hat{b}_{1}}^{2}+2 s_{\hat{b}_{1}, \hat{b}_{2}} X+s_{\hat{b}_{2}}^{2} X^{2}\right)}_{\text {first-order approximation }} \\
& +\underbrace{s_{a_{1}}^{2}\left(s_{\hat{b}_{1}}^{2}+2 s_{\hat{b}_{1}, \hat{b}_{2}} X+s_{\hat{b}_{2}}^{2} X^{2}\right)}_{\text {additional part from second-order approximation (exact variance) }} \\
= & \left(\hat{b}_{1}+\hat{b}_{2} X\right)^{2} s_{\hat{a}_{1}}^{2}+\left(\hat{a}_{1}^{2}+s_{\hat{a}_{1}}^{2}\right)\left(s_{\hat{b}_{1}}^{2}+2 s_{\hat{b}_{1}, \hat{b}_{2}} X+s_{\hat{b}_{2}}^{2} X^{2}\right)
\end{aligned}
$$


Model 2: When the $a$ Path Is Moderated by $W$

$$
\begin{aligned}
\hat{\boldsymbol{\theta}}= & {\left[\begin{array}{lll}
\hat{a}_{1} & \hat{a}_{3} & \hat{b}_{1}
\end{array}\right]^{\prime} \quad f(\hat{\boldsymbol{\theta}} \mid W)=\hat{b}_{1}\left(\hat{a}_{1}+\hat{a}_{3} W\right) } \\
\hat{\mathbf{\Sigma}}(\hat{\boldsymbol{\theta}})= & {\left[\begin{array}{ccc}
s_{\hat{a}_{1}}^{2} & s_{\hat{a}_{1}, \hat{a}_{3}} & 0 \\
s_{\hat{a}_{1}, \hat{a}_{3}} & s_{\hat{a}_{3}}^{2} & 0 \\
0 & 0 & s_{\hat{b}_{1}}^{2}
\end{array}\right] \quad \mathbf{H}=\left[\begin{array}{ccc}
0 & 0 & 1 \\
0 & 0 & W \\
1 & W & 0
\end{array}\right] } \\
\operatorname{var}[f(\hat{\boldsymbol{\theta}} \mid W)] \approx & \mathbf{D}^{\prime} \hat{\boldsymbol{\Sigma}}(\hat{\boldsymbol{\theta}}) \mathbf{D}+\frac{1}{2} \operatorname{tr}\left\{(\mathbf{H} \hat{\boldsymbol{\Sigma}}(\hat{\boldsymbol{\theta}}))^{2}\right\} \\
= & \underbrace{\left(\hat{a}_{1}+\hat{a}_{3} W\right)^{2} s_{\hat{b}_{1}}^{2}+\hat{b}_{1}^{2}\left(s_{\hat{a}_{1}}^{2}+2 s_{\hat{a}_{1}, \hat{a}_{3}} W+s_{\hat{a}_{3}}^{2} W^{2}\right)}_{\text {first-order approximation }} \\
& +\underbrace{s_{\hat{b}_{1}}^{2}\left(s_{\hat{a}_{1}}^{2}+2 s_{\hat{a}_{1}, \hat{a}_{3}} W+s_{\hat{a}_{3}}^{2} W^{2}\right)}_{\text {additional part from second-order approximation (exact variance) }} \\
= & \left(\hat{a}_{1}+\hat{a}_{3} W\right)^{2} s_{\hat{b}_{1}}^{2}+\left(\hat{b}_{1}^{2}+s_{\hat{b}_{1}}^{2}\right)\left(s_{\hat{a}_{1}}^{2}+2 s_{\hat{a}_{1}, \hat{a}_{3}} W+s_{\hat{a}_{3}}^{2} W^{2}\right)
\end{aligned}
$$

Model 3: When the $b$ Path Is Moderated by $W$

$$
\begin{aligned}
\hat{\boldsymbol{\theta}}= & {\left[\begin{array}{lll}
\hat{a}_{1} & \hat{b}_{1} & \hat{b}_{3}
\end{array}\right]^{\prime} \quad f(\hat{\boldsymbol{\theta}} \mid W)=\hat{a}_{1}\left(\hat{b}_{1}+\hat{b}_{3} W\right) } \\
\hat{\boldsymbol{\Sigma}}(\hat{\boldsymbol{\theta}})= & {\left[\begin{array}{ccc}
s_{\hat{a}_{1}}^{2} & 0 & 0 \\
0 & s_{\hat{b}_{1}}^{2} & s_{\hat{b}_{1}, \hat{b}_{3}} \\
0 & s_{\hat{b}_{1}, \hat{b}_{3}} & s_{\hat{b}_{3}}^{2}
\end{array}\right] \quad \mathbf{H}=\left[\begin{array}{ccc}
0 & 1 & W \\
1 & 0 & 0 \\
W & 0 & 0
\end{array}\right] } \\
\operatorname{var}[f(\hat{\boldsymbol{\theta}} \mid W)] \approx & \mathbf{D}^{\prime} \hat{\boldsymbol{\Sigma}}(\hat{\boldsymbol{\theta}}) \mathbf{D}+\frac{1}{2} \operatorname{tr}\left\{(\mathbf{H} \hat{\boldsymbol{\Sigma}}(\hat{\boldsymbol{\theta}}))^{2}\right\} \\
= & \underbrace{\left(\hat{b}_{1}+\hat{b}_{3} W\right)^{2} s_{\hat{a}_{1}}^{2}+\hat{a}_{1}^{2}\left(s_{\hat{b}_{1}}^{2}+2 s_{\hat{b}_{1}, \hat{b}_{3}} W+s_{\hat{b}_{3}}^{2} W^{2}\right)}_{\text {first-order approximation }} \\
& +\underbrace{s_{\hat{a}_{1}}^{2}\left(s_{\hat{b}_{1}}^{2}+2 s_{\hat{b}_{1}, \hat{b}_{3}} W+s_{\hat{b}_{3}}^{2} W^{2}\right)}_{\text {additional part from second-order approximation (exact variance) }} \\
= & \left(\hat{b}_{1}+\hat{b}_{3} W\right)^{2} s_{\hat{a}_{1}}^{2}+\left(\hat{a}_{1}^{2}+s_{\hat{a}_{1}}^{2}\right)\left(s_{\hat{b}_{1}}^{2}+2 s_{\hat{b}_{1}, \hat{b}_{3}} W+s_{\hat{b}_{3}}^{2} W^{2}\right)
\end{aligned}
$$


Model 4: When the $a$ Path is Moderated by $W$ and the $b$

Path Is Moderated by $Z$

$$
\begin{gathered}
\hat{\boldsymbol{\theta}}=\left[\begin{array}{llll}
\hat{a}_{1} & \hat{a}_{3} & \hat{b}_{1} & \hat{b}_{3}
\end{array}\right]^{\prime} \quad f(\hat{\boldsymbol{\theta}} \mid W, Z)=\left(\hat{a}_{1}+\hat{a}_{3} W\right)\left(\hat{b}_{1}+\hat{b}_{3} Z\right) \\
\hat{\boldsymbol{\Sigma}}(\hat{\boldsymbol{\theta}})=\left[\begin{array}{cccc}
s_{\hat{a}_{1}}^{2} & s_{\hat{a}_{1}, \hat{a}_{3}} & 0 & 0 \\
s_{\hat{a}_{1}, \hat{a}_{3}} & s_{\hat{a}_{3}}^{2} & 0 & 0 \\
0 & 0 & s_{\hat{b}_{1}}^{2} & s_{\hat{b}_{1}, \hat{b}_{3}} \\
0 & 0 & s_{\hat{b}_{1}, \hat{b}_{3}} & s_{\hat{b}_{3}}^{2}
\end{array}\right] \quad \mathbf{H}=\left[\begin{array}{cccc}
0 & 0 & 1 & Z \\
0 & 0 & W & W Z \\
1 & W & 0 & 0 \\
Z & W Z & 0 & 0
\end{array}\right] \\
\operatorname{var}[f(\hat{\boldsymbol{\theta}} \mid W, Z)] \approx \\
= \\
\mathbf{D}^{\prime} \hat{\boldsymbol{\Sigma}}(\hat{\boldsymbol{\theta}}) \mathbf{D}+\frac{1}{2} \operatorname{tr}\left\{(\mathbf{H} \hat{\boldsymbol{\Sigma}}(\hat{\boldsymbol{\theta}}))^{2}\right\} \\
+\underbrace{\left(\hat{a}_{1}+\hat{a}_{3} W\right)^{2}\left(s_{\hat{b}_{1}}^{2}+2 s_{\hat{b}_{1}, \hat{b}_{3}} Z+s_{\hat{b}_{3}}^{2} Z^{2}\right)}_{\text {first-order approximation }} \\
+\underbrace{\left(s_{\hat{b}_{1}}^{2}+2 \hat{b}_{\hat{b}_{1}, \hat{b}_{3}} Z+\hat{b}_{3} Z\right)^{2}\left(s_{\hat{a}_{1}}^{2}+2 s_{\hat{a}_{1}, \hat{a}_{3}} W+s_{\hat{a}_{3}}^{2} Z^{2}\right)}_{\text {additional part from second-order approximation (exact variance) }}
\end{gathered}
$$

Model 5: When the $a$ and $b$ Paths Are Both Moderated by $W$

$$
\begin{gathered}
\hat{\boldsymbol{\theta}}=\left[\begin{array}{llll}
\hat{a}_{1} & \hat{a}_{3} & \hat{b}_{1} & \hat{b}_{2}
\end{array}\right]^{\prime} \quad f(\hat{\boldsymbol{\theta}} \mid W)=\left(\hat{a}_{1}+\hat{a}_{3} W\right)\left(\hat{b}_{1}+\hat{b}_{2} W\right) \\
\hat{\boldsymbol{\Sigma}}(\hat{\boldsymbol{\theta}})=\left[\begin{array}{cccc}
s_{\hat{a}_{1}}^{2} & s_{\hat{a}_{1}, \hat{a}_{3}} & 0 & 0 \\
s_{\hat{a}_{1}, \hat{a}_{3}} & s_{\hat{a}_{3}}^{2} & 0 & 0 \\
0 & 0 & s_{\hat{b}_{1}}^{2} & s_{\hat{b}_{1}, \hat{b}_{2}} \\
0 & 0 & s_{\hat{b}_{1}, \hat{b}_{2}} & s_{\hat{b}_{2}}^{2}
\end{array}\right] \quad \mathbf{H}=\left[\begin{array}{cccc}
0 & 0 & 1 & W \\
0 & 0 & W & W^{2} \\
1 & W & 0 & 0 \\
W & W^{2} & 0 & 0
\end{array}\right] \\
\operatorname{var}[f(\hat{\boldsymbol{\theta}} \mid W)] \approx \\
=\underbrace{\mathbf{D}^{\prime} \hat{\boldsymbol{\Sigma}}(\hat{\boldsymbol{\theta}}) \mathbf{D}+\frac{1}{2} \operatorname{tr}\left\{(\mathbf{H} \hat{\boldsymbol{\Sigma}}(\hat{\boldsymbol{\theta}}))^{2}\right\}}_{\text {first-order approximation }} \\
= \\
+\underbrace{\left(\hat{b}_{1}+\hat{b}_{2} W\right)^{2}\left(s_{\hat{a}_{1}}^{2}+2 s_{\hat{a}_{1}, \hat{a}_{3}} W+s_{\hat{a}_{3}}^{2} W^{2}\right)}_{\text {additional part from second-order approximation (exact variance) }}
\end{gathered}
$$

